\title{
NUMERICAL COMPARISONS OF TWO LONG-WAVE LIMIT MODELS
}

\author{
Stéphane LabBÉ ${ }^{1}$ And Lionel Paumond ${ }^{1}$
}

\begin{abstract}
The Benney-Luke equation (BL) is a model for the evolution of three-dimensional weakly nonlinear, long water waves of small amplitude. In this paper we propose a nearly conservative scheme for the numerical resolution of (BL). Moreover, it is known (Paumond, Differential Integral Equations 16 (2003) 1039-1064; Pego and Quintero, Physica D 132 (1999) 476-496) that (BL) is linked to the Kadomtsev-Petviashvili equation for almost one-dimensional waves propagating in one direction. We study here numerically the link between (KP) and (BL) and we point out the coupling effects emerging by considering two solitary waves propagating in two opposite directions.
\end{abstract}

Mathematics Subject Classification. 35L05, 35Q51, 35Q53, 65J15, 65M70.

Received: October 3, 2003.

\section{INTRODUCTION}

The difficulties met to work with the full water-wave problem lead to derive simplified model in the special case of long waves. The model equation we will consider describe the evolution of three-dimensional weakly nonlinear, long water waves of small amplitude,

$$
\Phi_{t t}-\Delta \Phi+\mu\left(a \Delta^{2} \Phi-b \Delta \Phi_{t t}\right)+\varepsilon\left(\Phi_{t} \Delta \Phi+(\nabla \Phi)_{t}^{2}\right)=0
$$

where $\Phi(x, y, t)$ is the velocity potential on the bottom of the domain after rescaling the variables and $\nabla$ and $\Delta$ are respectively the two-dimensional gradient and Laplacian. The constants $a$ and $b$ are positive and such that $a-b=\sigma-\frac{1}{3} \neq 0$ where $\sigma$ is the Bond number. $\varepsilon$ is the amplitude parameter (nonlinearity coefficient) and $\mu=\left(h_{0} / L\right)^{2}$ is the long wave parameter (dispersion coefficient), where $h_{0}$ is the equilibrium depth and $L$ is the length scale. This equation was first derived by Benney and Luke (BL) (see [5]) when $a=1 / 6$ and $b=1 / 2$ with no surface tension. Let us remark that the model (1) is not valid for $a=b(\sigma=1 / 3)$ and in this case, it corresponds to a nonlinear wave equation with no dispersion. Precisely, in this special case, (BL) is not linearly well-posed. Then, we can derive (see [14]) an equation still valid when we suppose that $\sigma$ is equal or close to $1 / 3$,

$$
\Phi_{t t}-\Delta \Phi+\sqrt{\varepsilon}\left[a \Delta^{2} \Phi-b \Delta \Phi_{t t}\right]+\varepsilon\left[B \Delta^{2} \Phi_{t t}-A \Delta^{3} \Phi\right]+\varepsilon\left[2 \nabla \Phi_{t} \cdot \nabla \Phi+\Phi_{t} \Delta \Phi\right]=0,
$$

where $\varepsilon=\mu^{2}$ and the positive parameters $A, B$ are linked. This model becomes linearly well-posed even if $\sigma$ is close or equal to $1 / 3$. For the study of the Cauchy problem for these nonlinear equations we infer to $[14,15]$.

Keywords and phrases. Benney-Luke, Kadomtsev-Petviashvili, spectral method, long wave limit.

1 Laboratoire de Mathématiques, Université Paris-Sud, Bâtiment 425, 91405 Orsay Cedex, France.

e-mail: stephane.labbe@math.u-psud.fr 
Putting a solution in (1) having the form $\Phi(x, y, t)=f(X, Y, T)$ with the scaling $X=x-t, Y=\sqrt{\varepsilon} y$ and $T=\varepsilon t / 2$, we obtain at the lowest order in $\varepsilon($ see $[12,16])$

$$
\left(u_{T}-\left(\sigma-\frac{1}{3}\right) u_{X X X}+3 u u_{X}\right)_{X}+u_{Y Y}=0
$$

where $u=f_{X}$. We recall that if $\sigma>1 / 3$, this equation is KP-I, if $\sigma<1 / 3$, it is KP-II. By setting $a-b=$ $\sigma-1 / 3=\theta \mu$ with $\theta \in \mathbb{R}$ independent of $\mu$ and doing exactly the same in (2) we obtain the fifth order KP-I equation (see [14])

$$
\partial_{X}\left(\partial_{T} u-\theta \partial_{X}^{3} u+\frac{1}{45} \partial_{X}^{5} u+3 u \partial_{X} u\right)+\partial_{Y}^{2} u=0
$$

where we have set $u=f_{X}$. Let us remark that if we suppose $f$ not depending on the $y$ variable we obtain respectively the KdV equation and the Kawahara equation.

The family of KP equations can formally be derived from the full water-wave problem (see [1]). A rigorous proof of this derivation is known in the one dimensional case (see $[8,10,17])$ and consistency results for the two dimensional problem can be found in $[7,11]$. It is quite natural to link rigorously KP to intermediate models, that is done in $[4,9]$ and specially in $[14,15](1)$ and (2) are considered.

From a numerical point of view, Milewski and Keller compared in [12] doubly periodic solutions of BL to those of KP but the algorithm they give is only able to compute these particular solutions. Milewski and Tabak (see [13]) solved the BL equation and related models using a pseudospectral method based on an explicit Runge-Kutta time discretization, but they do not give a mathematical study of this scheme. In particular they simulate the propagation of a single solitary wave and the interaction of two solitary waves at small angle. In [6], Berger and Milewski derive formally lump solitons to a generalized Benney-Luke equation from those of KP, they also study numerically the collision of such waves and their propagation over an obstacle.

The purpose of our work is to compare the KP dynamic to that of the BL one thanks to a simple and nearly conservative scheme in order to solve equations (1). Moreover we give a rigorous numerical analysis of our method.

Precisely, in [15], it is shown that if $u=u(X, Y, T)$ is an enough regular solution on $\left[0, T_{0}\right] \times \mathbb{R}^{2}$ of the $\mathrm{KP}$ equation

$$
\left(u_{T}-(a-b) u_{X X X}+3 u u_{X}\right)_{X}+u_{Y Y}=0,
$$

where $X=x-t, Y=\varepsilon^{\frac{1}{2}} y, T=\varepsilon t / 2$, then there exists $\varepsilon_{0}>0$, and a constant $C_{0}>0$ such that for every $\left.\varepsilon \in] 0, \varepsilon_{0}\right]$, the BL equation has a unique solution $\Phi$ in $\left[0,2 T_{0} / \varepsilon\right] \times \mathbb{R}^{2}$ of the form

$$
\Phi(x, y, t)=\partial_{X}^{-1} u\left(x-t, \varepsilon^{1 / 2} y, \varepsilon t / 2\right)+\varepsilon \rho\left(x, \varepsilon^{1 / 2} y, t\right),
$$

where $\rho(\cdot, \cdot, 0)=\rho_{t}(\cdot, \cdot, 0)=0$, satisfying

$$
\sup _{t \in\left[0,2 T_{0} / \varepsilon\right]}\left\|\Phi_{t}(x, y, t)+u\left(x-t, \varepsilon^{1 / 2} y, \varepsilon t / 2\right)\right\|_{L^{2}\left(\mathbb{R}_{x, y}^{2}\right)} \leq C \varepsilon^{3 / 4},
$$

with $C$ independent of $\varepsilon$ but depending on $u$. The solutions $u$ of KP considered in [15], are localized on $\mathbb{R}^{2}$, moreover, we have made some restrictive assumptions on $\partial_{X}^{-1} u(X, Y, T)\left(\in H^{p}\left(\mathbb{R}^{2}\right)\right.$ for some large $\left.p\right)$. Lannes proves in [11], starting from a Boussinesq system, that these assumptions can be removed, and then, the rate of convergence is decreasing with the loss of zero mass assumptions. In this paper, we will illustrate Theorem 1.1 of [15], when $u$ is a line soliton or a periodic lump soliton of KP which do not satisfy these assumptions. We show that the rate of convergence seems to be at least $3 / 4$ in these special cases.

Moreover, in [15], we have only considered the approximation of BL by one KP equation. In $[3,4,17]$ the case of two waves propagating in two opposite directions is considered. Precisely, in [4], the authors have shown two convergence theorems between the solutions of a general hyperbolic system, and two systems of KP equations coupled or uncoupled. In the uncoupled case, the convergence obtained by their method is of order $o(1)$, and of 
order $\mathcal{O}(\varepsilon)$ in the coupled one. Then we could think that the coupling effects determine the order of convergence. In this paper, we show up this coupling effect numerically by estimating the error between a BL solution by two solitary waves of two uncoupled KP equations. The numerical results leads us to surmise that the rate of convergence is not worse in this case.

This work is organized as follows. In a first section, we describe the numerical scheme used to solve BL and we give its mathematical study. Next, we show the numerical results corresponding to a bench. In Section 4, we compare BL and KP for two kind of solitary waves solutions of KP. The last section is devoted to the study of two waves propagating in two opposite directions.

\section{THE NUMERICAL SCHEME}

In this section, we present the discretization of periodic solutions of (1); in the following, we will work on the box $\Omega_{L M}=[0,2 L] \times[0,2 M]$.

We will proceed in two steps: a finite difference discretization in time and a spectral discretization in the space directions.

\subsection{Transformation of the Benney-Luke equation}

In order to discretize system (1), we set, for every $T$ in $\mathbb{R}^{+}, s \geq 2$, and $\Phi$ in $C^{0}\left([0, T], H^{s-2}\left(\Omega_{L M}\right)\right)$

$$
U=\left(\begin{array}{c}
\mathcal{F}(\Phi) \\
\mathcal{F}\left(\Phi_{t}\right)
\end{array}\right)=\left(\begin{array}{c}
U_{1} \\
U_{2}
\end{array}\right)
$$

where, for every $\varphi$ in $L^{2}\left(\Omega_{L M}\right)$ and $\xi$ in $Z_{L M}=\left\{(l \pi / L, m \pi / M),(l, m) \in \mathbb{Z}^{2}\right\}$,

$$
\mathcal{F}(\varphi)(\xi)=\int_{\Omega_{L M}} \varphi(x) \mathrm{e}^{-i x \cdot \xi} \mathrm{d} x .
$$

Let us set for every $\xi \in Z_{L M}, Q_{a}(\xi)=\left(1+\mu a|\xi|^{2}\right), Q_{b}(\xi)=\left(1+\mu b|\xi|^{2}\right), Q(\xi)=\frac{Q_{a}(\xi)}{Q_{b}(\xi)}|\xi|^{2}$ and $A(\xi)=$ $\left(\begin{array}{cc}0 & 1 \\ Q(\xi) & 0\end{array}\right)$. Using these notations, equation (1) can be rewritten

$$
\forall \xi \in Z_{L M}, U_{t}(\xi)=A(\xi) U(\xi)+\mathcal{N}(U, U, \xi)
$$

where

$$
\mathcal{N}(U, V, \xi)=\left(0,-\varepsilon Q_{b}^{-1}(\xi) \mathcal{F}\left[\mathcal{F}^{-1}\left(U_{2}\right) \mathcal{F}^{-1}\left(-|\xi|^{2} V_{1}\right)+2\left(\mathcal{F}^{-1}\left(i \xi_{1} U_{1}\right) \mathcal{F}^{-1}\left(i \xi_{1} V_{2}\right)+\mathcal{F}^{-1}\left(i \xi_{2} U_{1}\right) \mathcal{F}^{-1}\left(i \xi_{2} V_{2}\right)\right)\right]\right)^{t}
$$

\subsection{The time discretization}

First of all, we discretize in the direction of time. One of our goal is to have a good preservation for the approximation of the quantity

$$
\mathcal{S}(U)=\left\|U_{2}\right\|_{L^{2}\left(\Omega_{L M}\right)}^{2}+\mu b\left\|i \xi U_{2}\right\|_{L^{2}\left(\Omega_{L M}\right)}^{2}+\left\|i \xi U_{1}\right\|_{L^{2}\left(\Omega_{L M}\right)}^{2}+\mu a\left\||\xi|^{2} U_{1}\right\|_{L^{2}\left(\Omega_{L M}\right)}^{2} .
$$

Indeed, it is not difficult to see that this quantity is conserved by the dynamic of (7) if $U$ is periodic on $\Omega_{L M}$ (see [15] for the case of the whole space). The equation to solve, rewritten in its integral form, is

$$
\forall\left(t_{0}, t\right) \in[0, T], t_{0}<t, U(t)-U\left(t_{0}\right)=\int_{t_{0}}^{t}(A(\xi) U(s)+\mathcal{N}(U(s), U(s), \xi)) \mathrm{d} s .
$$


The discrete space considered will be the space of the piece-wise linear functions in time taking their values in $H^{s-2}\left(\Omega_{L M}\right)$ for $s \geq 4$. Let $\mathcal{W}_{1}=\left\{U \in \mathcal{S}^{\prime}\left(\Omega_{L M}\right) \mid \mathcal{F}^{-1}(U) \in C^{0}\left([0, T], H^{s-2}\left(\Omega_{L M}\right)\right) \times C^{0}\left([0, T], H^{s-2}\left(\Omega_{L M}\right)\right)\right\}$ for $s \geq 4$, we set $k$ a time-step and $N$ the greatest integer such that $N k \leq T$, so we define the discrete time space

$$
\mathcal{W}_{1, k}^{t}=\left\{U \in \mathcal{W}_{1} \mid \forall i \in\{0, \ldots, N-1\}, U \chi_{[i k,(i+1) k]}(t) \in\left(\mathbb{P}^{1}(\mathbb{C}, \mathbb{R})\right)^{2}\right\}
$$

where $\chi_{I}$, for $I \subset \mathbb{R}$, is the characteristic function of $I$ and $\mathbb{P}^{1}(\mathbb{C}, \mathbb{R})$ designates the set of polynoms on $\mathbb{R}$ of degree lesser or equal to one with complex coefficients. So, we define the projection $\mathrm{P}_{k}^{t}$ from $\mathcal{W}_{1}$ onto $\mathcal{W}_{1, k}^{t}$ and its transposed operator $\mathrm{P}_{k}^{*, t}$ by

$$
\begin{aligned}
& \forall U \in \mathcal{W}_{1}, \mathrm{P}_{k}^{t}(U)=\frac{1}{k} \sum_{i=0}^{N-1}(U(i k)((i+1) k-t)+U((i+1) k)(t-i k)) \chi_{[i k,(i+1) k]}(t), \\
& \forall V \in \mathcal{W}_{1, k}^{t}, \mathrm{P}_{k}^{*, t}(V)=V
\end{aligned}
$$

We consider the following approximation of (9), that is find $V \in \mathcal{W}_{1, k}^{t}$ such that:

$$
\begin{aligned}
& V^{0}=U^{0}, \\
& \forall i \in\{0, . ., N-1\}, V^{i+1}-V^{i}=\int_{i k}^{(i+1) k}(A(\xi) V(s)+\mathcal{N}(V(s), V(s), \xi)) \mathrm{d} s,
\end{aligned}
$$

where, for all $i$ in $\{0, \ldots, N\}, V^{i}=V(i k)$.

\subsubsection{An approximated scheme}

A first "natural" approximation is to use a quadrature formula to integrate (10): a one point Gauss quadrature formula. This method "gives" the classical Cranck-Nicholson scheme

$$
V^{i+1}=V^{i}+k\left(A(\xi)\left(\frac{V^{i+1}+V^{i}}{2}\right)+\mathcal{N}\left(\frac{V^{i+1}+V^{i}}{2}, \frac{V^{i+1}+V^{i}}{2}, \xi\right)\right) .
$$

By convergence of the quadrature formula used, we deduce the consistency of the scheme. The stability, in sense of the semi-norm $S(U)$ is directly deduced from the fact that for all $i$ in $\{0, \ldots, N-1\}, S\left(U^{i+1}\right)-S\left(U^{i}\right)=0$. This property is proved by multiplying the second line (11) by $Q_{b}(\xi)$ and after by $\frac{V^{i+1}+V^{i}}{2}$ (we use the same principle of demonstration than the one used in [15] in the regular case). Using this result, we deduce that the scheme (11) is well-posed and of order 2. Even if this scheme is conservative, we will use the following exact time integration of (10) in order to have a better time approximation.

\subsubsection{The exact scheme}

Now, using the fact that $\mathcal{N}(U, V, \xi)$ is bi-linear in $(U, V)$, we can integrate (10) and obtain

$$
\begin{aligned}
\forall i \in\{0, . ., N-1\}, V^{i+1}-V^{i}= & k \int_{0}^{1}\left((1-s) A(\xi)\left(V^{i+1}\right)+s A(\xi)\left(V^{i}\right)\right. \\
& +(1-s)^{2} \mathcal{N}\left(V^{i+1}, V^{i+1}, \xi\right)+s^{2} \mathcal{N}\left(V^{i}, V^{i}, \xi\right) \\
& \left.+s(1-s)\left(\mathcal{N}\left(V^{i+1}, V^{i}, \xi\right)+\mathcal{N}\left(V^{i}, V^{i+1}, \xi\right)\right)\right) \mathrm{d} s \\
= & \frac{k}{2} A(\xi)\left(V^{i+1}+V^{i}\right)+\frac{k}{3}\left(\mathcal{N}\left(V^{i}, V^{i}, \xi\right)+\mathcal{N}\left(V^{i+1}, V^{i+1}, \xi\right)\right. \\
& \left.+\frac{1}{2}\left(\mathcal{N}\left(V^{i+1}, V^{i}, \xi\right)+\mathcal{N}\left(V^{i}, V^{i+1}, \xi\right)\right)\right)
\end{aligned}
$$


The time discretization can be written, for all $i$ in $\{0, . ., N-1\}$ and for $V^{0}=U^{0}$,

$$
\begin{aligned}
V^{i+1}= & V^{i}+k A(\xi)\left(\frac{V^{i+1}+V^{i}}{2}\right) \\
& +\frac{2 k}{3}\left(\frac{\mathcal{N}\left(V^{i}, V^{i}, \xi\right)+\mathcal{N}\left(V^{i+1}, V^{i+1}, \xi\right)}{2}+\mathcal{N}\left(\frac{V^{i+1}+V^{i}}{2}, \frac{V^{i+1}+V^{i}}{2}, \xi\right)\right) .
\end{aligned}
$$

\subsection{The space discretization: spectral discretization}

The spectral discretization is the projection of functions on a finite number of frequencies

$$
\forall(i, j) \in\left\{-\frac{N_{x}}{2}, \ldots, \frac{N_{x}}{2}-1\right\} \times\left\{-\frac{N_{y}}{2}, \ldots, \frac{N_{y}}{2}-1\right\}, \xi_{i, j}=\left(i \frac{\pi}{L}, j \frac{\pi}{M}\right)
$$

We set $h=\left(\frac{L}{N_{x}}, \frac{M}{N_{y}}\right)$.

\subsubsection{Finite Fourier transform}

We set

$$
\begin{aligned}
\mathcal{W}_{1, h}^{x} & =\left(\left\{u \mid \mathcal{F}^{-1}(u) \in \operatorname{Span}\left\{\mathrm{e}^{i x \cdot \xi_{n, p}}, \forall(n, p) \in\left\{-\frac{N_{x}}{2}, \ldots, \frac{N_{x}}{2}-1\right\} \times\left\{-\frac{N_{y}}{2}, \ldots, \frac{N_{y}}{2}-1\right\}\right\}\right\}\right)^{2}, \\
\mathcal{W}_{1, k, h} & =\left\{U \in \mathcal{W}_{1, h}^{x} \mid \forall i \in\{0, \ldots, N-1\}, U \chi_{[i k,(i+1) k]}(t) \in\left(\mathbb{P}^{1}(\mathbb{C}, \mathbb{R})\right)^{2}\right\}
\end{aligned}
$$

then we define $\mathrm{P}_{h}^{x}$, the projection in sense of $\mathrm{L}^{2}\left(\Omega_{L M}\right)$ from $H^{s-2}\left(\Omega_{L M}\right) \times H^{s-2}\left(\Omega_{L M}\right)$ into $\mathcal{W}_{1, h}^{x}$. We remark that $\mathcal{W}_{1, h}^{x}$ is include in $H^{s-2}\left(\Omega_{L M}\right) \times H^{s-2}\left(\Omega_{L M}\right)$, that means that the transposed operator of $\mathrm{P}_{h}^{x}$ is the identity on $\mathcal{W}_{1, h}^{x}$. So we can define

$$
\forall v \in H^{s-2}\left(\Omega_{L M}\right) \times H^{s-2}\left(\Omega_{L M}\right), \quad \mathcal{F}_{h}(v)=\mathcal{F}\left(\mathrm{P}_{h}^{x}(v)\right) .
$$

$\mathcal{F}\left(\mathcal{W}_{1, h}^{x}\right)$ is isomorph to $\mathbb{R}^{\left(N_{x}+N_{y}\right)} ;$ for each element $u$ of $\mathcal{F}\left(\mathcal{W}_{1, h}^{x}\right)$ we associate an element $U$ of $\mathbb{R}^{\left(N_{x}+N_{y}\right)}$

$$
\forall(n, p) \in\left\{-\frac{N_{x}}{2}, \ldots, \frac{N_{y}}{2}-1\right\} \times\left\{-\frac{N_{y}}{2}, \ldots, \frac{N_{y}}{2}-1\right\}, U_{n, p}=\int_{\Omega_{L M}} u \mathrm{e}^{-i x \cdot \xi_{n, p}} \mathrm{~d} x
$$

and for each element $U$ of $\mathbb{R}^{\left(N_{x}+N_{y}\right)}$ we associate an element $u$ of $\mathcal{F}\left(\mathcal{W}_{1, h}^{x}\right)$

$$
\forall x \in \Omega_{L M}, \quad \mathcal{F}^{-1}(u)(x)=\sum_{n=-\frac{N_{x}}{2}}^{\frac{N_{x}}{2}-1} \sum_{p=-\frac{N_{y}}{2}}^{\frac{N_{y}}{2}-1} U_{n, p} \mathrm{e}^{-i x \cdot \xi_{n, p}}=\tilde{U}(x) .
$$

So we can define a finite Fourier transform

$$
\forall U \in \mathbb{R}^{\left(N_{x}+N_{y}\right)}, \quad \mathcal{F}_{h}^{-1}(U)=\sum_{n=-\frac{N_{x}}{2}}^{\frac{N_{x}}{2}-1} \sum_{p=-\frac{N_{y}}{2}}^{\frac{N_{y}}{2}-1} U_{n, p} \mathcal{F}\left(\mathrm{e}^{-i x \cdot \xi_{n, p}}\right) .
$$


1.3.2. The time-space discretization

The time-space discretization can be written, for all $i$ in $\{0, . ., N-1\}$ and for $V^{0}=\mathrm{P}_{h}^{x}\left(U^{0}\right)$,

$$
\begin{aligned}
V^{i+1}= & V^{i}+k A_{h}(\xi)\left(\frac{V^{i+1}+V^{i}}{2}\right) \\
& +\frac{2}{3}\left(\frac{\mathcal{N}_{h}\left(V^{i}, V^{i}, \xi\right)+\mathcal{N}_{h}\left(V^{i+1}, V^{i+1}, \xi\right)}{2}+\mathcal{N}_{h}\left(\frac{V^{i+1}+V^{i}}{2}, \frac{V^{i+1}+V^{i}}{2}, \xi\right)\right),
\end{aligned}
$$

where

$$
\forall U \in \mathcal{W}_{1, h}^{x}, A_{h}(\xi)(U)=\mathcal{F}_{h}\left(\mathcal{F}^{-1}\left(\left(\mathrm{P}_{h}^{x}(A(\xi) \mathcal{F}(\tilde{U})(\xi))\right)\right)\right.
$$

and

$$
\forall(U, V) \in\left(\mathcal{W}_{1, h}^{x}\right)^{2}, \mathcal{N}_{h}(U, V, \xi)=\mathcal{F}_{h}\left(\mathcal{F}^{-1}\left(\mathrm{P}_{h}^{x}(\mathcal{N}(\mathcal{F}(\tilde{U})(\xi), \mathcal{F}(\tilde{V})(\xi), \xi))\right)\right)
$$

\section{Convergence And order of the exact scheme}

We will now prove the consistence and stability of (13).

Theorem 2.1. The scheme (13) is consistent. There exists a positive real constant $C$, only depending of the solution on (1) such that the consistency error epsilon satisfy for every $i$ in $\{0, \ldots, N-1\}$ :

$$
\left\|\eta_{i}\right\|_{l^{2}\left(\Omega_{h}\right)} \leq \frac{\varepsilon k^{5 / 2}}{|\tilde{h}|^{2}} C
$$

where $\frac{1}{|\tilde{h}|^{2}}=\frac{N_{x}^{2}}{L^{2}}+\frac{N_{y}^{2}}{M^{2}}$.

Proof. We set, for $\left(u^{i}\right)_{i=0, \ldots, N-1}$ solution of (13):

$$
\forall i \in\{0, \ldots, N-1\}, \forall t \in\left[t_{i}, t_{i+1}\right], u_{k}^{i}(t)=\frac{t-t_{i}}{k} u^{i+1}+\frac{t_{i+1}-t}{k} u^{i},
$$

and for all $v$ in $C^{0}\left(\left[t_{i}, t_{i+1}\right]\right)$ and $i$ in $\{0, \ldots, N-1\}$,

$$
\mathcal{I}_{i}(v)(t)=\frac{t-t_{i}}{k} v\left(t_{i+1}\right)+\frac{t_{i+1}-t}{k} v\left(t_{i}\right) .
$$

Setting $u=\left(\phi, \phi_{t}\right)^{t}$ solution of $(1)$, for all $i$ in $\{0, \ldots, N-1\}$, we have

$$
\begin{aligned}
\eta_{i} & =u\left(t_{i+1}\right)-u\left(t_{i}\right)-\int_{t_{i}}^{t_{i+1}}\left(A(\xi) \mathcal{I}_{i}(u)(t)+\mathcal{N}\left(\mathcal{I}_{i}(u)(t), \mathcal{I}_{i}(u)(t), \xi\right)\right) \mathrm{d} t \\
& =\int_{t_{i}}^{t_{i+1}}\left(\begin{array}{l}
u_{2}(t)-\mathcal{I}_{i}\left(u_{2}\right)(t) \\
Q(\xi)\left(u_{1}(t)-\mathcal{I}_{i}\left(u_{1}\right)(t)\right)+\mathcal{N}_{2}(u(t), u(t), \xi)-\mathcal{N}_{2}\left(\mathcal{I}_{i}(u)(t), \mathcal{I}_{i}(u)(t), \xi\right)
\end{array}\right) \mathrm{d} t .
\end{aligned}
$$

Therefore, using the fact that $u_{1}$ is an element of $C^{3}\left([0, T], L^{2}\left(\Omega_{L M}\right)\right)$, we use the classical polynomial interpolation errors to prove that

$$
\left\|u_{2}(t)-\mathcal{I}_{i}\left(u_{2}\right)(t)\right\|_{L^{2}\left(\Omega_{L M}\right)} \leq \frac{k^{5 / 2}}{2 \sqrt{30}}\left\|u_{2}^{(2)}\right\|_{L^{\infty}\left([0, T] ; L^{2}\left(\Omega_{L M}\right)\right)},
$$




$$
\begin{aligned}
\| Q(\xi)\left(u_{1}(t)-\right. & \left.\mathcal{I}_{i}\left(u_{1}\right)(t)\right)\left\|_{L^{2}\left(\Omega_{L M}\right)} \leq \frac{k^{5 / 2}}{2|\tilde{h}|^{2} \sqrt{30}}\right\| u_{1}^{(2)} \|_{L^{\infty}\left([0, T] ; L^{2}\left(\Omega_{L M}\right)\right)}, \\
\| \mathcal{N}_{2}(u(t), u(t), \xi)- & \mathcal{N}_{2}\left(\mathcal{I}_{i}(u)(t), \mathcal{I}_{i}(u)(t), \xi\right) \|_{L^{2}\left(\Omega_{L M}\right)} \leq \\
& \frac{3 \varepsilon k^{7 / 2}}{2 \sqrt{30}|\tilde{h}|^{2}}\left[\frac{k^{5 / 2}}{2 \sqrt{30}}\left\|u_{1}^{(2)}\right\|_{L^{\infty}\left([0, T] ; L^{2}\left(\Omega_{L M}\right)\right)}\left\|u_{1}^{(2)}\right\|_{L^{\infty}\left([0, T] ; L^{2}\left(\Omega_{L M}\right)\right)}\right. \\
& +\left\|u_{2}\right\|_{L^{\infty}\left([0, T] ; L^{2}\left(\Omega_{L M}\right)\right)}\left\|u_{1}^{(2)}\right\|_{L^{\infty}\left([0, T] ; L^{2}\left(\Omega_{L M}\right)\right)} \\
& \left.+\left\|u_{1}\right\|_{L^{\infty}\left([0, T] ; L^{2}\left(\Omega_{L M}\right)\right)}\left\|u_{2}^{(2)}\right\|_{L^{\infty}\left([0, T] ; L^{2}\left(\Omega_{L M}\right)\right)}\right]
\end{aligned}
$$

where $\mathcal{N}_{2}$ is the second component of $\mathcal{N}$, so, by summation of these inequalities, we obtain the conclusion.

Remark 2.1. The proof of consistency for the approximated scheme is the same. The difference is in the evaluation of the nonlinear term consistency, for Cranck Nicholson, a term of quadrature error so instead of $k^{7 / 2}$, we find out $k^{3}$. Numerically, this estimation is confirmed by the fact that the exact scheme is more accurate than the Cranck Nicholson scheme.

Using the fact that the Cranck Nicholson scheme is stable, we prove that the exact scheme is stable.

Theorem 2.2. For $\frac{\varepsilon k}{|\hat{h}|^{2}}<1$, the scheme (13) is stable.

Proof. We use the notations introduced above. We set $\left(u^{i}\right)_{i=0, \ldots, N-1}$ solution of $(11),\left(v^{i}\right)_{i=0, \ldots, N-1}$ solution of (13) and $w^{i}=v^{i}-u^{i}$ for every $i$ in $\{0, \ldots, N\}$; so $\left(v^{i}\right)_{i=0, \ldots, N-1}$ is solution of

$$
\begin{aligned}
w^{i+1}-w^{i}= & -\int_{t_{i}}^{t_{i+1}}\left(A(\xi) v_{k}^{i}(t)+\mathcal{N}\left(v_{k}^{i}(t), v_{k}^{i}(t), \xi\right)\right) \mathrm{d} t \\
& +k A(\xi) u_{k}^{i}\left(\frac{t_{i}+t_{i+1}}{2}\right)+\mathcal{N}\left(v_{k}^{i}\left(\frac{t_{i}+t_{i+1}}{2}\right), v_{k}^{i}\left(\frac{t_{i}+t_{i+1}}{2}\right), \xi\right)
\end{aligned}
$$

using the Peano error formula, we obtain

$$
\begin{aligned}
w^{i+1}-w^{i}= & k A(\xi) \frac{w^{i}+w^{i+1}}{2}+k \frac{w^{i}+w^{i+1}}{2}(1,0)^{t} \\
& +(0,1)^{t} \int_{t_{i}}^{t_{i+1}} \mathcal{N}\left(w_{k}^{i}(t), w_{k}^{i}(t), \xi\right) \mathrm{d} t-(0,1)^{t} \frac{7}{12} \mathcal{N}\left(u^{i+1}-u^{i}, u^{i+1}-u^{i}, \xi\right) .
\end{aligned}
$$

Then, using a Grönwall discrete inequality, assuming that $\frac{\varepsilon k}{|\grave{h}|^{2}}<1$ and that $\left(w^{i}\right)_{i=0, \ldots, N-1}$ is controlled by $k$ and $\left(u^{i}\right)_{i=0, \ldots, N-1}$ with the fact that (11) is stable, we deduce that (13) is also stable because the distance between $u^{i}$ and $v^{i}$ is proved to be controlled by $k$.

\section{A NUMERICAL BENCH}

In this section, we will give some numerical results in order to validate our numerical scheme. The test we decide to do is the same as that of [13], that is the simulation of the evolution of a solitary wave. We know that for $a=0, b=1 / 3$, (no surface tension, $\sigma=0$ ) equation (1) possesses an explicit solitary wave solution having the form

and

$$
\Phi(x, y, t)=\frac{A}{c k} \tanh \left(k_{1} x+k_{2} y-c k t\right)
$$

$$
\Phi_{t}(x, y, t)=-A \operatorname{sech}^{2}\left(k_{1} x+k_{2} y-c k t\right)
$$




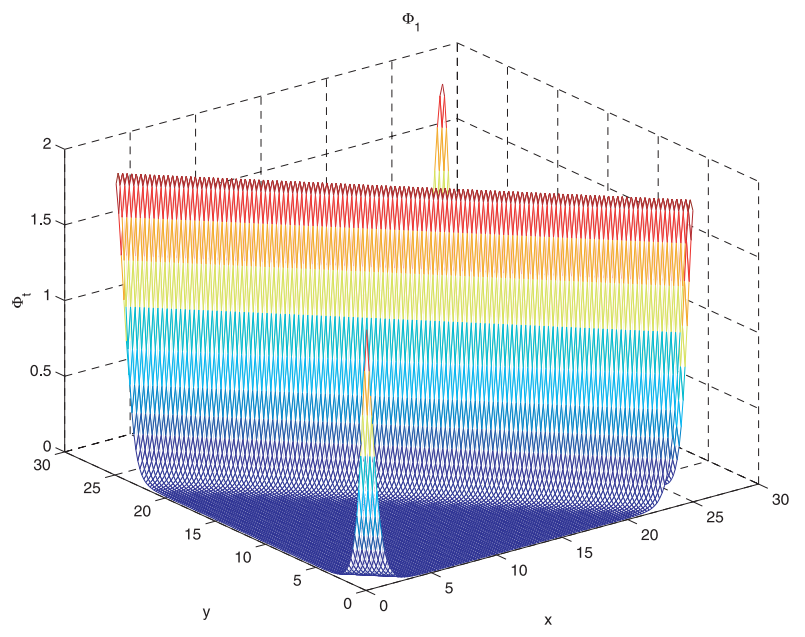

Figure 1. $\Phi_{1}$.

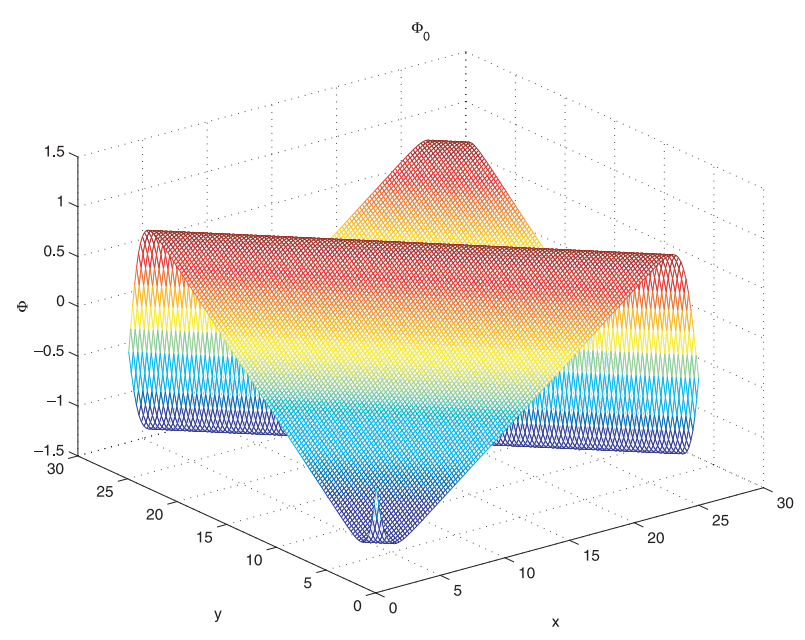

FiguRE 2. $\Phi_{0}$.

where $k=\sqrt{k_{1}^{2}+k_{2}^{2}}$. The amplitude $A$ and the celerity $c$ of the wave are linked by

$$
A=\frac{4}{3} c^{2} k^{2}
$$

Moreover, in $\mathbb{R}^{2}$, the following relation between $c$ and $k$ holds

$$
c^{2}=\frac{1}{1-\frac{4}{3} \varepsilon k^{2}}
$$

The numerical problem we consider is periodic in space, then we must fit the initial data in a periodic box $\Omega_{L M}=[0 ; 2 L] \times[0 ; 2 M]$ satisfying $k_{1} L=k_{2} M$ as follows

$$
\Phi(x, y, t)=\frac{A}{c k}\left[\tanh \left(k_{1} x+k_{2} y-c k t\right)-\frac{1}{k_{1} L} \tanh \left(k_{1} L\right)\left(k_{1} x+k_{2} y\right)\right],
$$

and

$$
\Phi_{t}(x, y, t)=-A \operatorname{sech}^{2}\left(k_{1} x+k_{2} y-c k t\right) .
$$

The relation between $A$ and $c$ remains unchanged but now we have

$$
c^{2}=\frac{1}{1-\frac{4}{3} \varepsilon k^{2}+4 \varepsilon k^{2} \frac{1}{k_{1} L} \tanh \left(k_{1} L\right)} .
$$

The initial data are the following

$$
\Phi_{0}(x, y)=\frac{A}{c k}\left[\tanh \left(k_{1} x+k_{2} y\right)-\frac{1}{k_{1} L}\left(k_{1} x+k_{2} y\right) \tanh \left(k_{1} L\right)\right],
$$

and

$$
\Phi_{1}(x, y)=-A \operatorname{sech}^{2}\left(k_{1} x+k_{2} y\right) .
$$

We give their shape in Figures 1 and 2 . 


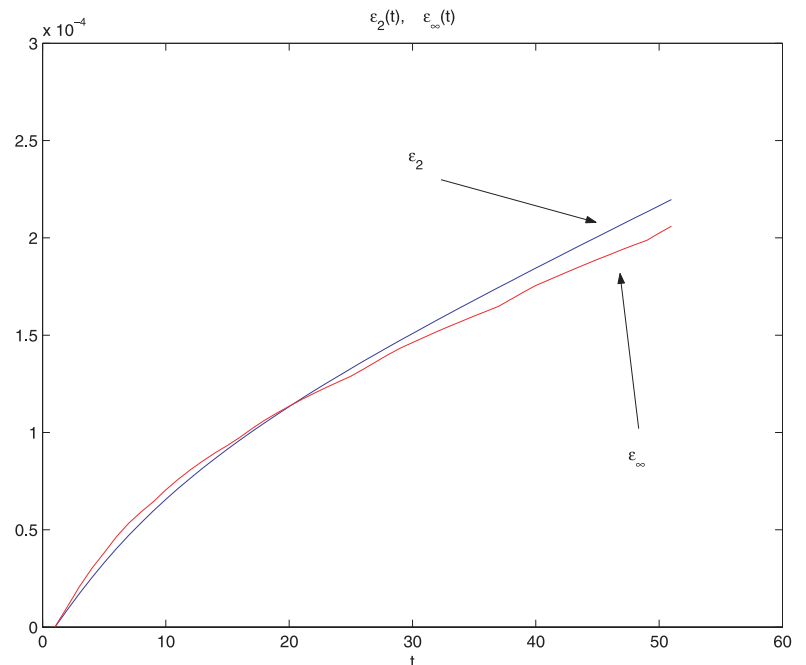

FIGURE 3. $256 \times 256$ points, $\Delta t=$ $0.005, t=0, \ldots, 50$.

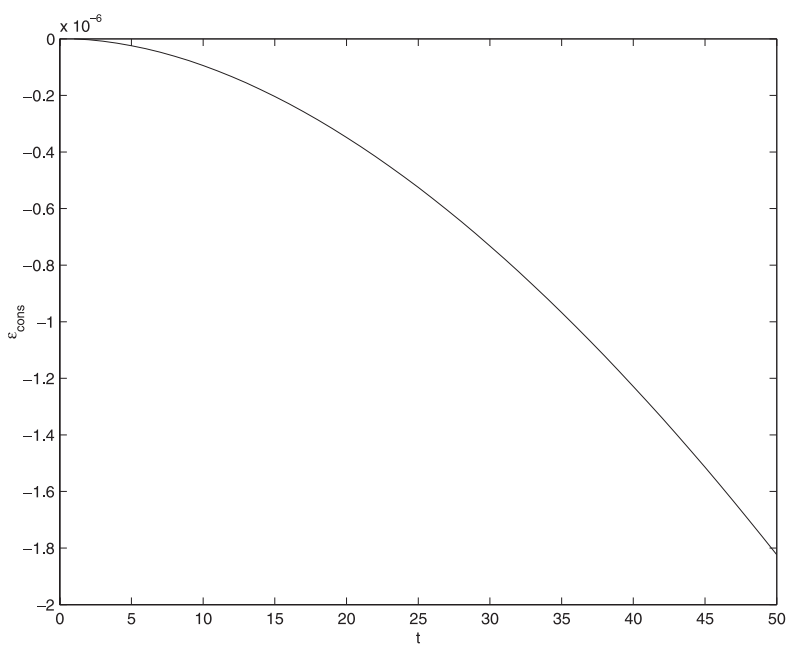

FigURE $5 . \mathcal{S}_{0}(\tilde{\Phi}): 256 \times 256$ points, $\Delta t=0.005, t=0, \ldots, 50$.

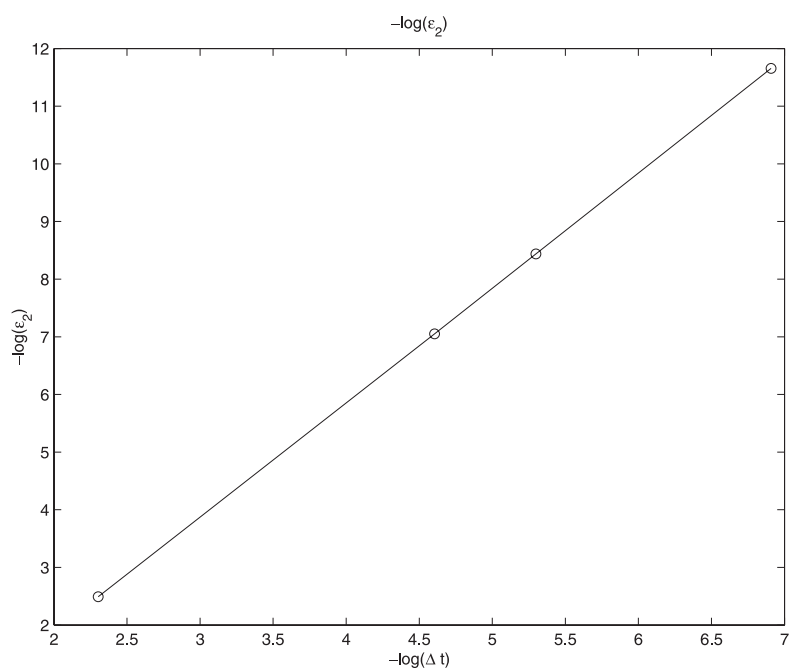

FiguRE $4.256 \times 256$ points, $t=50$.

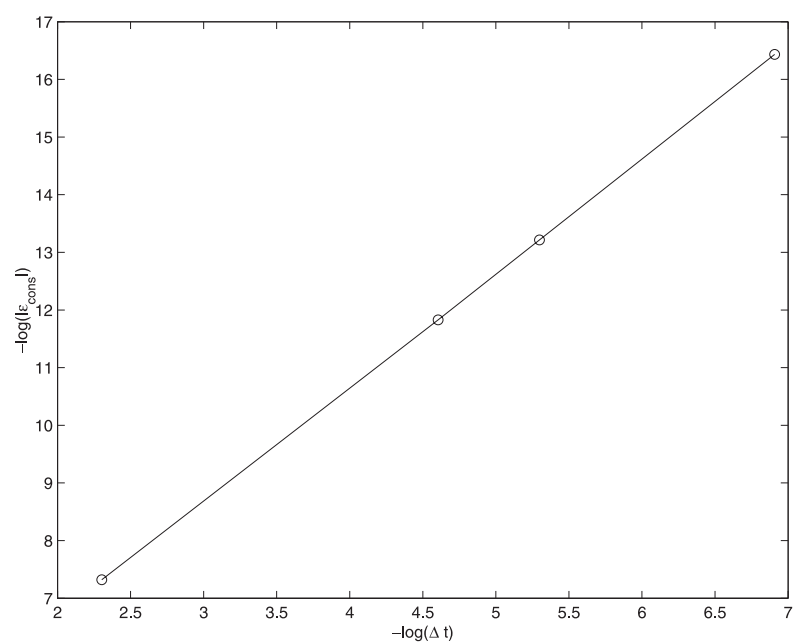

FiguRE $6 .-\log \left(\varepsilon_{\text {cons }}\right): 256 \times 256$ points, $t=50$.

Let us set $\varepsilon_{2}=\frac{\left\|\tilde{\Phi}_{t}-\Phi_{t}\right\|_{l^{2}\left(\Omega_{L M}\right)}}{\left\|\Phi_{t}\right\|_{l^{2}\left(\Omega_{L M}\right)}}, \varepsilon_{\infty}=\frac{\left\|\tilde{\Phi}_{t}-\Phi_{t}\right\|_{l^{\infty}\left(\Omega_{L M}\right)}}{\left\|\Phi_{t}\right\|_{l^{\infty}\left(\Omega_{L M}\right)}}$ and $\varepsilon_{\text {cons }}=\frac{\mathcal{S}_{0}(\tilde{\Phi})(t)-\mathcal{S}_{0}(\tilde{\Phi})(0)}{\mathcal{S}_{0}(\tilde{\Phi})(0)}$, where $\Phi_{t}$ is the exact solution given by (19), $\tilde{\Phi}_{t}$ is the numerical one and

$$
\mathcal{S}_{0}(\Phi)=\left\|\Phi_{t}\right\|_{L^{2}}^{2}+\|\nabla \Phi\|_{L^{2}}^{2}+\mu b\left\|\nabla \Phi_{t}\right\|_{L^{2}}^{2}+\mu a\|\Delta \Phi\|_{L^{2}}^{2} .
$$

We see in Figure 4 that the time convergence of our scheme is of order $(\Delta t)^{2}$. Figure 3 shows the evolution of the $l^{2}\left(\Omega_{L M}\right)$ relative error for $\Delta t=5 \times 10^{-3}$. These experiments have been done on a meshgrid of $256 \times 256$ points, corresponding to the following space steps: $\Delta x=0.17, \Delta y=0.34$.

Figure 5 points out that our scheme is dissipative for the energy $\mathcal{S}_{0}(\tilde{\Phi})$, but even for large $\Delta t($ e.g. $\Delta t=0.1)$, $\varepsilon_{\text {cons }}$ is less than $7 \times 10^{-4}$ (see Fig. 6). That confirms that our method is almost conservative and that the 


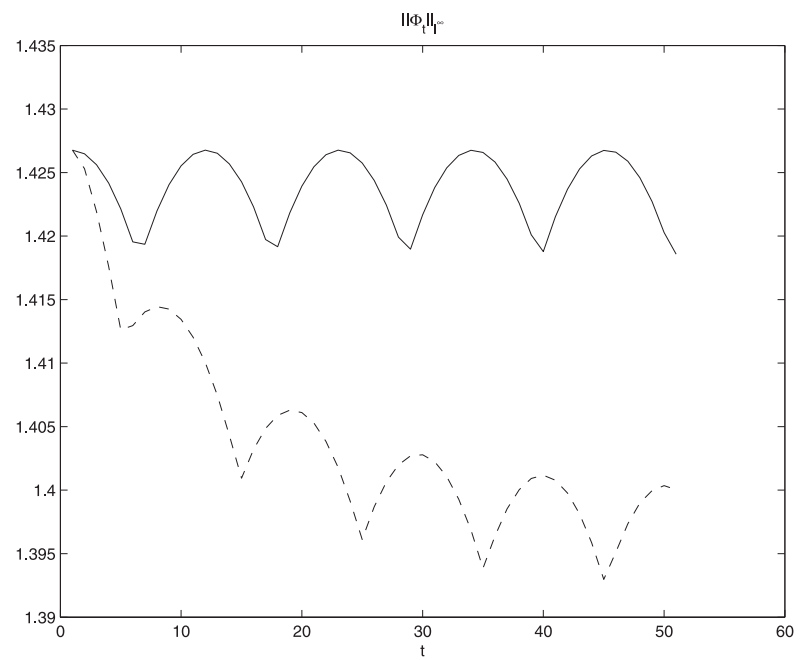

Figure $\quad 7 .\left\|\Phi_{t}\right\|_{l^{\infty}\left(\Omega_{L M}\right)} \quad$ (solid) and $\left\|\tilde{\Phi}_{t}\right\|_{l \infty\left(\Omega_{L M}\right)}$ (dashed): $256 \times$ 256 points, $\Delta t=0.1, t=0, \ldots, 50$.

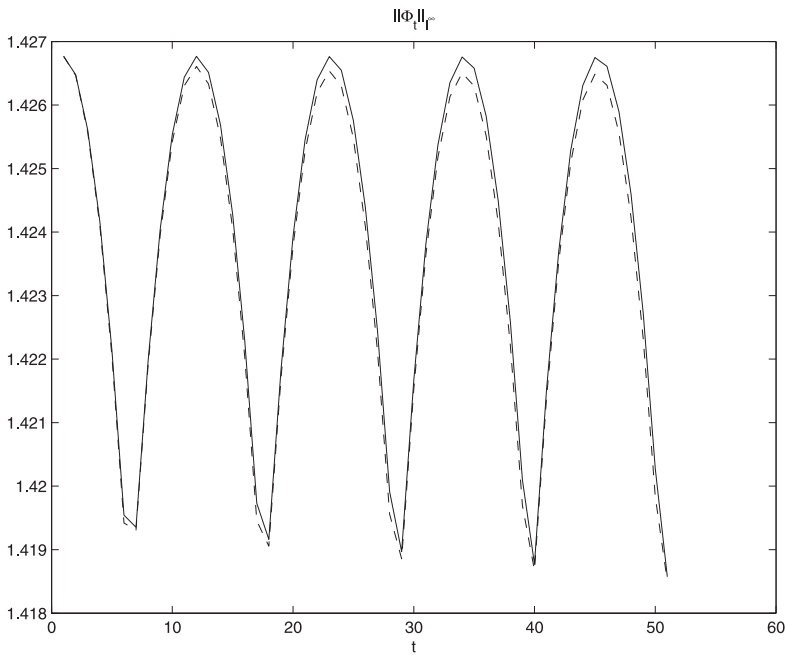

Figure 8. $\left\|\Phi_{t}\right\|_{l^{\infty}\left(\Omega_{L M}\right)} \quad$ (solid) and $\left\|\tilde{\Phi}_{t}\right\|_{l^{\infty}\left(\Omega_{L M}\right)}$ (dashed): $256 \times$ 256 points, $\Delta t=0.01, \quad t=$ $0, \ldots, 50$.

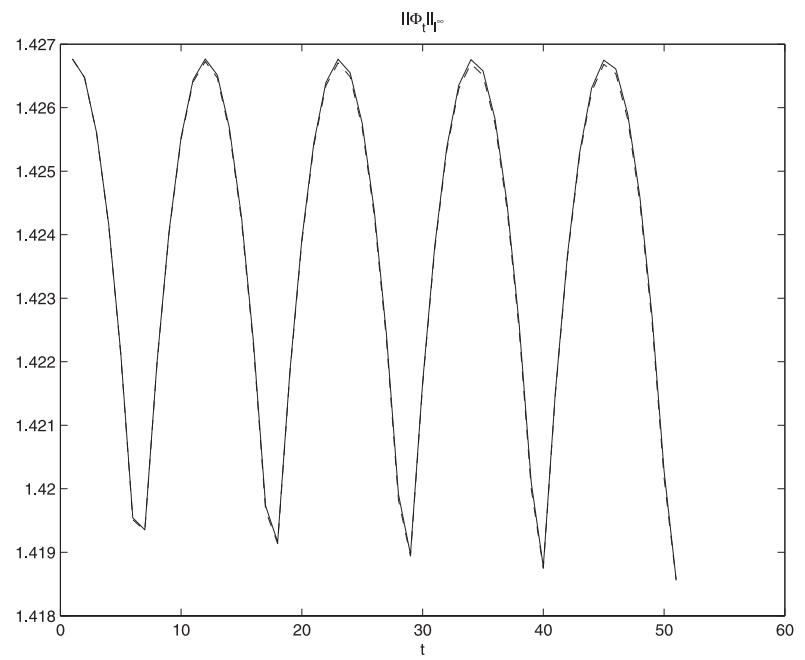

Figure 9. $\left\|\Phi_{t}\right\|_{l \infty\left(\Omega_{L M}\right)} \quad$ (solid) and $\left\|\tilde{\Phi}_{t}\right\|_{l^{\infty}\left(\Omega_{L M}\right)}$ (dashed): $256 \times$ 256 points, $\Delta t=0.005, t=$ $0, \ldots, 50$.

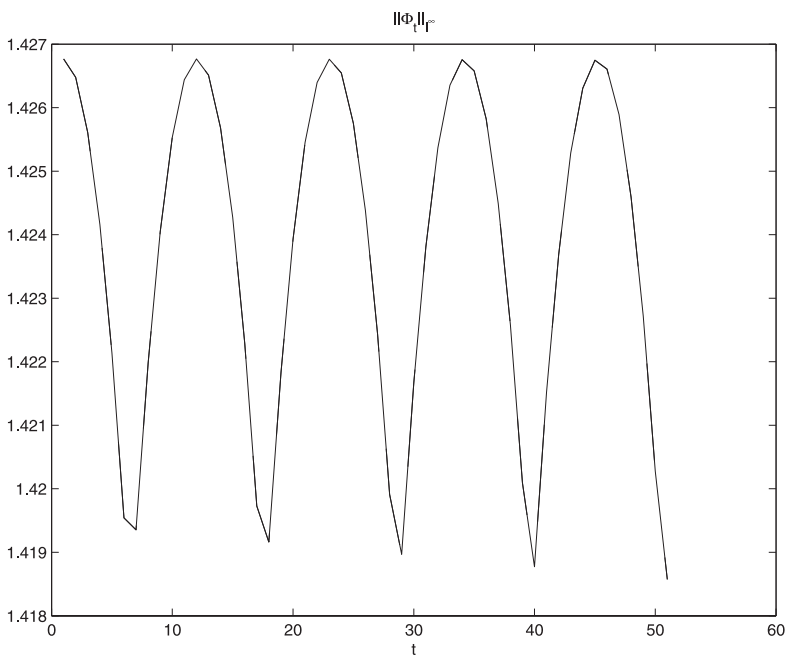

Figure 10. $\left\|\Phi_{t}\right\|_{l^{\infty}\left(\Omega_{L M}\right)}$ (solid) and $\left\|\tilde{\Phi}_{t}\right\|_{l^{\infty}\left(\Omega_{L M}\right)}$ (dashed): $256 \times$ 256 points, $\Delta t=0.001, t=$ $0, \ldots, 50$.

conservation is of order 2 in time. In Figures $7-10$, we compare the evolution of $\left\|\Phi_{t}\right\|_{l^{\infty}\left(\Omega_{L M}\right)}$ and $\left\|\tilde{\Phi}_{t}\right\|_{l^{\infty}\left(\Omega_{L M}\right)}$ for various time steps. The difference becomes virtually undistinguishable when $\Delta t=10^{-3}$, but for large time step we observe the stiffness of the problem. 


\section{Numerical COMPARISONS}

\subsection{Comparison with a KP line soliton}

In this section, we want to compare numerically two particular solutions of the KP and BL equations. Let take a line soliton of the KP equation

$$
\left(u_{T}-(a-b) u_{X X X}+3 u u_{X}\right)_{X}+u_{Y Y}=0
$$

having the form

$$
u(X, Y, T)=A \operatorname{sech}^{2}\left(k_{1} X+k_{2} Y-\omega T\right),
$$

with $A=-4(a-b) k_{1}^{2}, \omega=\frac{k_{2}^{2}-4(a-b) k_{1}^{4}}{k_{1}}$. Let us remark that

$$
\partial_{X}^{-1} u(X, Y, T)=\frac{A}{k_{1}} \tanh \left(k_{1} X+k_{2} Y-\omega T\right),
$$

does not satisfy all the hypothesis of Theorem 1.1 in [15]. We build our initial data from this solution,

$$
\begin{aligned}
& \Phi_{0}(x, y)=\frac{A}{k_{1}} \tanh \left(k_{1} x+k_{2} \varepsilon^{1 / 2} y\right) \\
& \Phi_{1}(x, y)=-\frac{A}{k_{1}}\left(k_{1}+\frac{\varepsilon}{2} \omega\right) \operatorname{sech}^{2}\left(k_{1} x+k_{2} \varepsilon^{1 / 2} y\right)
\end{aligned}
$$

and we fit them in a periodic box for the numerical experiments (see [13])

$$
\begin{aligned}
& \Phi_{0}(x, y)=\frac{A}{k_{1}}\left[\tanh \left(k_{1} x+k_{2} \varepsilon^{1 / 2} y\right)-\frac{1}{k_{1} L} \tanh \left(k_{1} L\right)\left(k_{1} x+k_{2} \varepsilon^{1 / 2} y\right)\right], \\
& \Phi_{1}(x, y)=-\frac{A}{k_{1}}\left(k_{1}+\frac{\varepsilon}{2} \omega\right) \operatorname{sech}^{2}\left(k_{1} x+k_{2} \varepsilon^{1 / 2} y\right) .
\end{aligned}
$$

Let us remark that it dictates to take $k_{1} L=k_{2} \varepsilon^{1 / 2} M$. The difficulty comes from the increasing of the box size when $\varepsilon$ tends to zero: $L=4 \pi, M_{\varepsilon}=\frac{4 \pi}{10 \sqrt{\varepsilon}}$, thus $\varepsilon$ must stay in a range fixed by the space step $\Delta y$. The parameters are chosen as follows: $k_{1}=1, k_{2}=10, a=2 / 3, b=1 / 3$ for KP-I (resp. $a=1 / 3, b=2 / 3$ for $\mathrm{KP}-\mathrm{II}), \Delta t=0.005$ and $128 \times 512$ points and $T_{0}=10^{-3}$.

The time $T_{0}$ corresponding to the existence of the KP soliton is chosen very tiny but the celerity $\omega$ of this soliton is of order 100. We give the numerical results for $\mathcal{E} r r_{\infty}=\sup _{\left[0,2 T_{0} / \varepsilon\right]}\left\|\Phi_{t}+u\right\|_{l \infty\left(\Omega_{L M_{\varepsilon}}\right)}$ in the following tabular. In the following $t_{\max }=2 T_{0} / \varepsilon$ will denote the final time for the computation.

\begin{tabular}{|c|c|c|c|c|c|c|c|}
\hline$\varepsilon$ & 0.01 & 0.005 & 0.001 & 0.0005 & 0.0001 & 0.00005 & 0.00004 \\
\hline$L$ & $4 \pi$ & $4 \pi$ & $4 \pi$ & $4 \pi$ & $4 \pi$ & $4 \pi$ & $4 \pi$ \\
\hline$M_{\varepsilon}$ & 12.6 & 17.8 & 39.7 & 56.2 & 125.7 & 177.7 & 198.7 \\
\hline$\Delta x$ & $1.96 \times 10^{-1}$ & $1.96 \times 10^{-1}$ & $1.96 \times 10^{-1}$ & $1.96 \times 10^{-1}$ & $1.96 \times 10^{-1}$ & $1.96 \times 10^{-1}$ & $1.96 \times 10^{-1}$ \\
\hline$\Delta y$ & $4.91 \times 10^{-2}$ & $6.94 \times 10^{-2}$ & $1.55 \times 10^{-1}$ & $2.19 \times 10^{-1}$ & $4.91 \times 10^{-1}$ & $6.94 \times 10^{-1}$ & $7.76 \times 10^{-1}$ \\
\hline$t_{\max }$ & 0.2 & 0.4 & 2 & 4 & 20 & 40 & 50 \\
\hline $\mathcal{E} r r_{\infty}(\mathrm{KP}-\mathrm{I})$ & $6.58 \times 10^{-1}$ & $3.29 \times 10^{-1}$ & $6.58 \times 10^{-2}$ & $3.29 \times 10^{-2}$ & $6.61 \times 10^{-3}$ & $3.44 \times 10^{-3}$ & $2.88 \times 10^{-3}$ \\
\hline $\mathcal{E} r r_{\infty}(\mathrm{KP}-\mathrm{II})$ & $6.76 \times 10^{-1}$ & $3.38 \times 10^{-1}$ & $6.76 \times 10^{-2}$ & $3.38 \times 10^{-2}$ & $6.89 \times 10^{-3}$ & $3.75 \times 10^{-3}$ & $3.21 \times 10^{-3}$ \\
\hline
\end{tabular}




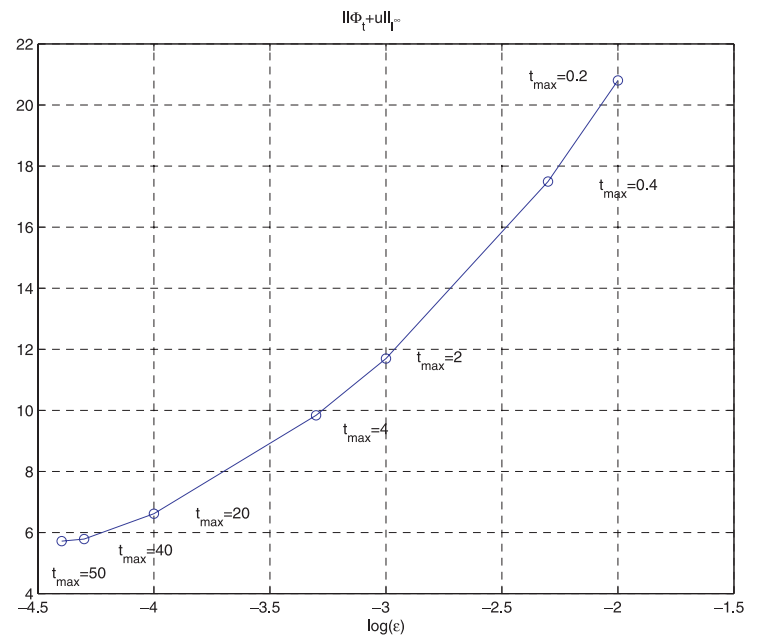

FiguRE 11. KP-I $\sup _{\left[0,2 T_{0} / \varepsilon\right]} \frac{\left\|\Phi_{t}+u\right\|_{l^{\infty}\left(\Omega_{L M_{\varepsilon}}\right)}}{\varepsilon^{3 / 4}}$ : $128 \times 512$ points, $\Delta t=0.005$.

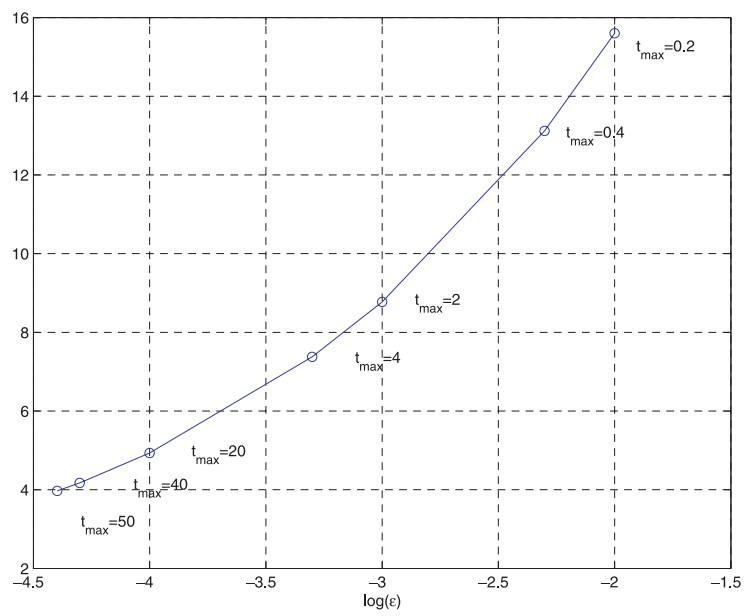

Figure 13. KP-I $\sup _{\left[0,2 T_{0} / \varepsilon\right]} \frac{\left\|\Phi_{t}+u\right\|_{l^{2}\left(\Omega_{L M_{\varepsilon}}\right)}}{\|u\|_{l^{2}\left(\Omega_{L M_{\varepsilon}}\right)} \varepsilon^{3 / 4}}$ : $128 \times 512$ points, $\Delta t=0.005$.

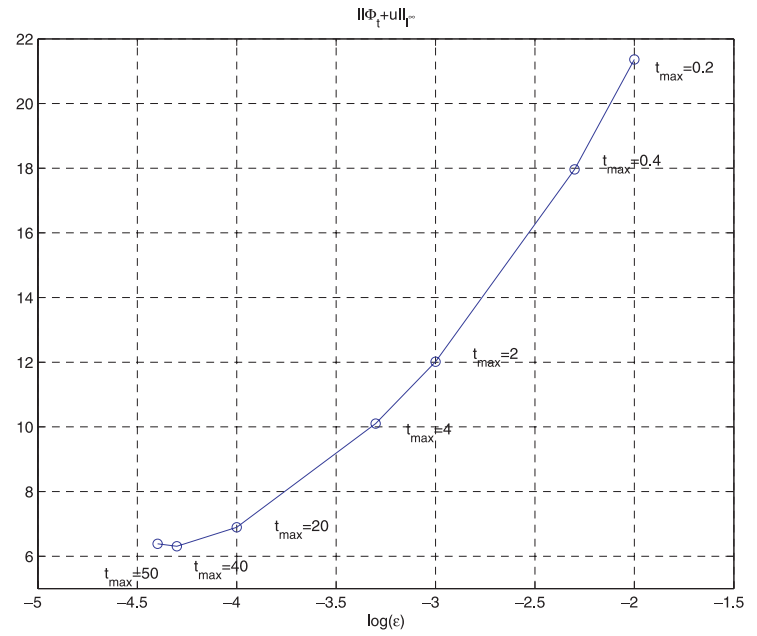

FigURE 12. KP-II $\sup _{\left[0,2 T_{0} / \varepsilon\right]} \frac{\left\|\Phi_{t}+u\right\|_{l^{\infty}\left(\Omega_{L M_{\varepsilon}}\right)}}{\varepsilon^{3 / 4}}$ : $128 \times 512$ points, $\Delta t=0.005$.

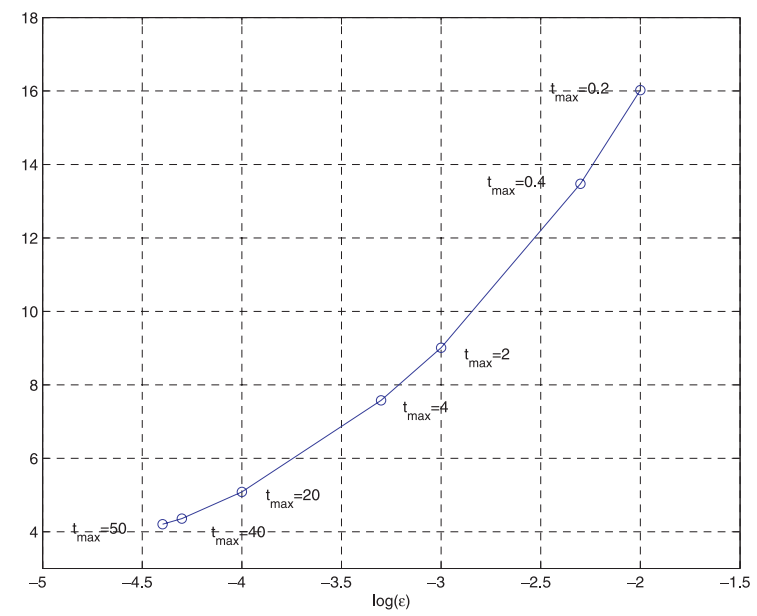

FiguRE 14. KP-II $\sup _{\left[0,2 T_{0} / \varepsilon\right]} \frac{\left\|\Phi_{t}+u\right\|_{l^{2}\left(\Omega_{L M_{\varepsilon}}\right)}}{\|u\|_{l^{2}\left(\Omega_{L M_{\varepsilon}}\right)} \varepsilon^{3 / 4}}$ : $128 \times 512$ points, $\Delta t=0.005$.

In (6), the constant $C$ depends on $\|u\|_{L^{2}\left(\mathbb{R}_{X, Y}^{2}\right)}$ but does not depend on $\varepsilon$. Since in our case this constant could depend on $\|u\|_{l^{2}\left(\Omega_{L M_{\varepsilon}}\right)}$ which is of order $1 / \varepsilon^{1 / 4}$ it is necessary to normalize by $\|u\|_{l^{2}\left(\Omega_{L M_{\varepsilon}}\right)}$. Thus

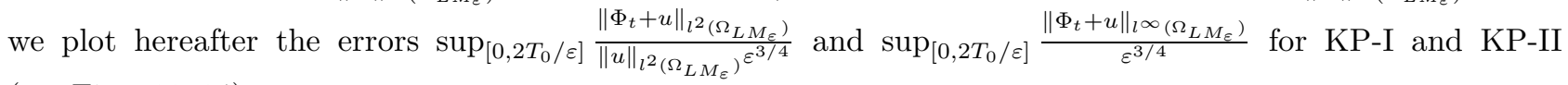
(see Figs. 11-14).

We observe that these functions decrease with $\varepsilon$ and it seems that they will be bounded by below, thus we can think that the rate $(3 / 4)$ of convergence is still valid in this case. 


\subsection{Periodic solitons of KP-I}

In this section we consider a class of soliton for KP-I (24) periodic in one direction and exponentially decreasing in the direction of propagation (see [2]),

$$
u(X, Y, T)=-4 \sqrt{a-b} \alpha^{2} \frac{1-\beta \cosh \left(\frac{\alpha X}{(a-b)^{1 / 4}}+\Omega(a-b)^{1 / 4} T\right) \cos (\delta Y)}{\left[\cosh \left(\frac{\alpha X}{(a-b)^{1 / 4}}+\Omega(a-b)^{1 / 4} T\right)-\beta \cos (\delta Y)\right]^{2}},
$$

where $\alpha$ and $\delta$ are fixed and $\Omega$ and $\beta$ are determined from $\beta=\sqrt{\frac{\delta^{2}-3 \alpha^{4}}{\delta^{2}}}$ and $\Omega=\frac{\delta^{2}+\alpha^{4}}{\alpha}$ with $\delta^{2}>3 \alpha^{4}$.

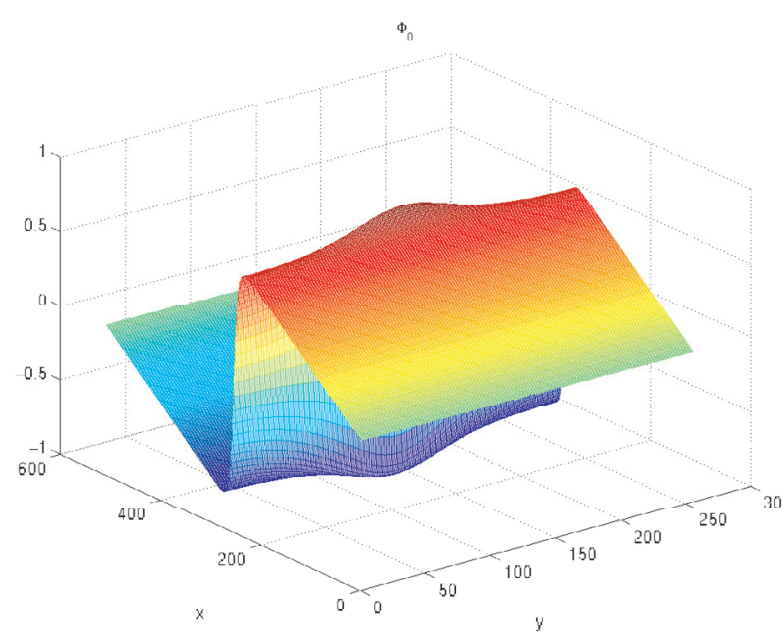

Figure 15. $\Phi_{0}$.

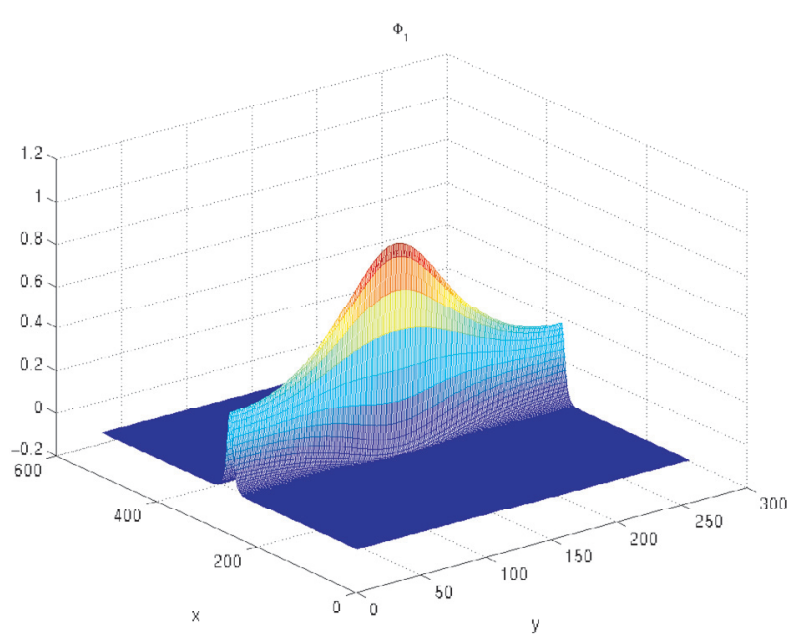

Figure 17. $\Phi_{1}$.

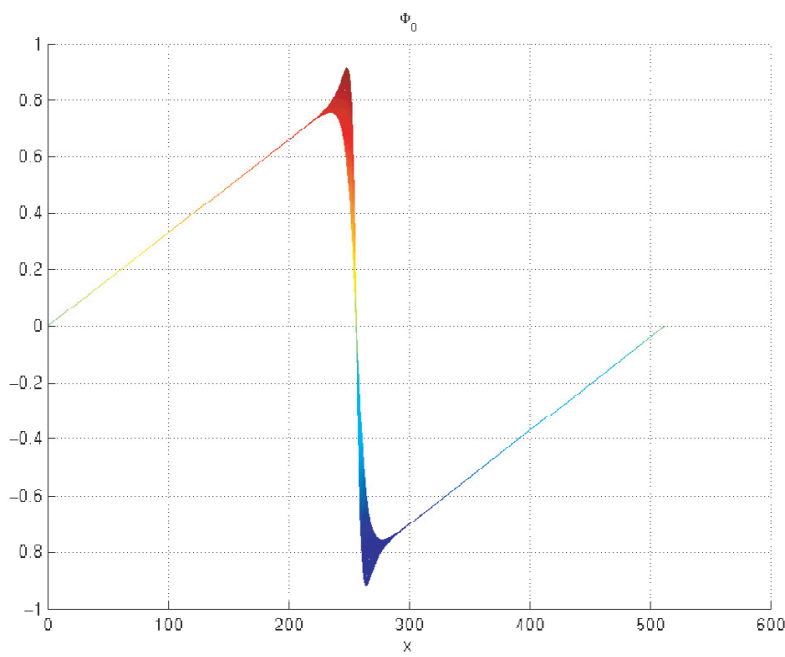

FiguRE 16. $\Phi_{0}$.

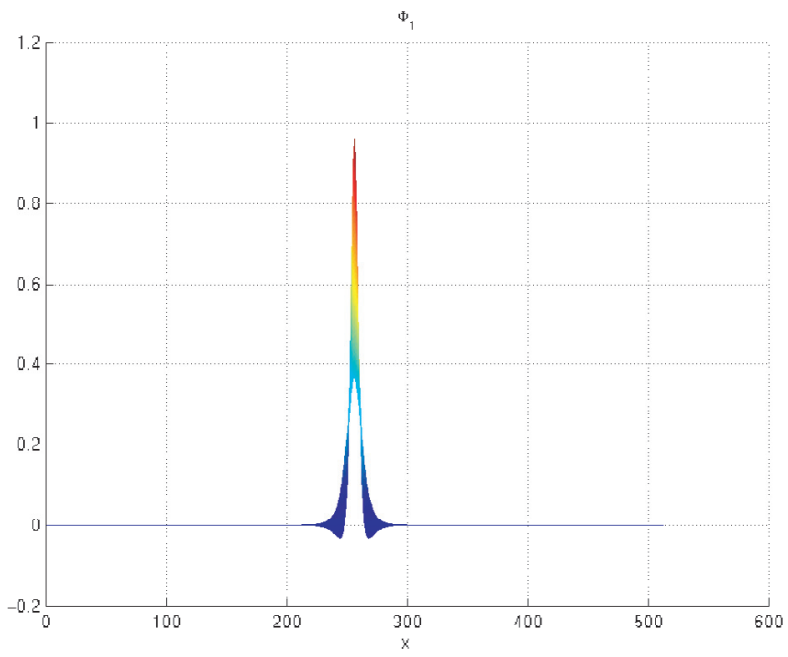

FiguRE $18 . \Phi_{1}$. 


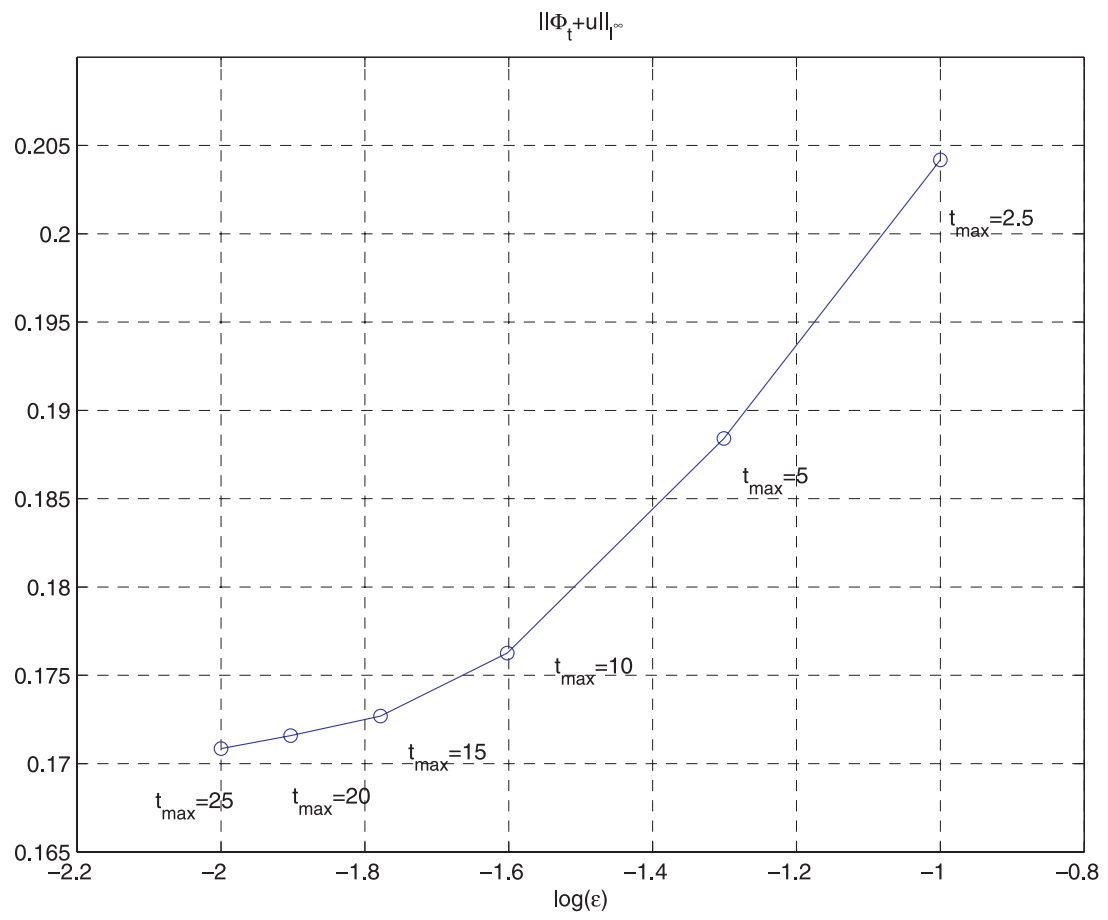

FIGURE 19. KP-I $\sup _{\left[0,2 T_{0} / \varepsilon\right]} \frac{\left\|\Phi_{t}+u\right\|_{l \infty\left(\Omega_{L M_{\varepsilon}}\right)}}{\varepsilon^{3 / 4}}: 128 \times 512$ points, $\Delta t=0.005$.

We build from (25) the initial data periodic in $x$ as follows,

$$
\Phi_{0}(x, y)=-8(a-b)^{3 / 4} \alpha\left[\frac{\tanh \left(\frac{\alpha x}{2(a-b)^{1 / 4}}\right)}{1-\beta \cos \left(\delta \varepsilon^{1 / 2} y\right)+\left[1+\beta \cos \left(\delta \varepsilon^{1 / 2} y\right)\right] \tanh ^{2}\left(\frac{\alpha L}{2(a-b)^{1 / 4}}\right)}+P(y) x\right]
$$

where

$$
P(y)=-\frac{\tanh \left(\frac{\alpha L}{2(a-b)^{1 / 4}}\right)}{1-\beta \cos \left(\delta \varepsilon^{1 / 2} y\right)+\left[1+\beta \cos \left(\delta \varepsilon^{1 / 2} y\right)\right] \tanh ^{2}\left(\frac{\alpha x}{2(a-b)^{1 / 4}}\right)},
$$

and

$$
\Phi_{1}(x, y)=\left[4 \sqrt{a-b} \alpha^{2}-2 \varepsilon(a-b) \alpha \Omega\right] \frac{1-\beta \cosh \left(\frac{\alpha x}{(a-b)^{1 / 4}}\right) \cos \left(\delta \varepsilon^{1 / 2} y\right)}{\left[\cosh \left(\frac{\alpha x}{(a-b)^{1 / 4}}\right)-\beta \cos \left(\delta \varepsilon^{1 / 2} y\right)\right]^{2}} .
$$

Let us remark that $\Phi_{0}$ and $\Phi_{1}$ are periodic in $y$ but we must choose $2 M_{\varepsilon}=\frac{2 \pi}{\delta \sqrt{\varepsilon}}$ (see Figs. 15-18). For our experiments we have chosen $T_{0}=0.125, N_{x}=512, N_{y}=256, L=70, M_{\varepsilon}=7 / \sqrt{\varepsilon}$, and we have ploted $\frac{\left\|\Phi_{t}+u\right\|_{l \infty\left(\Omega_{\left.L M_{\varepsilon}\right)}\right.}}{\varepsilon^{3 / 4}}$ for $\varepsilon=0.1,0.05,0.025,0.0167,0.0125,0.01$ (see Fig. 19). The results are almost the same as in the case of the line solitons, that is the $l^{\infty}$-error is decreasing and seems to be bounded from below by a positive constant. 


\begin{tabular}{|c|c|c|c|c|c|c|}
\hline$\varepsilon$ & 0.1 & 0.05 & 0.025 & 0.0167 & 0.0125 & 0.01 \\
\hline$L$ & 70 & 70 & 70 & 70 & 70 & 70 \\
\hline$M_{\varepsilon}$ & 22.14 & 31.30 & 44.27 & 54.22 & 62.6 & 70 \\
\hline$\Delta x$ & $2.73 \times 10^{-1}$ & $2.73 \times 10^{-1}$ & $2.73 \times 10^{-1}$ & $2.73 \times 10^{-1}$ & $2.73 \times 10^{-1}$ & $2.73 \times 10^{-1}$ \\
\hline$\Delta y$ & $1.73 \times 10^{-1}$ & $2.44 \times 10^{-1}$ & $3.46 \times 10^{-1}$ & $4.24 \times 10^{-1}$ & $4.89 \times 10^{-1}$ & $5.47 \times 10^{-1}$ \\
\hline$t_{\max }$ & 2.5 & 5 & 10 & 15 & 20 & 25 \\
\hline $\mathcal{E} r r_{\infty}$ & $3.63 \times 10^{-2}$ & $1.99 \times 10^{-2}$ & $1.11 \times 10^{-2}$ & $8.01 \times 10^{-3}$ & $6.41 \times 10^{-3}$ & $5.40 \times 10^{-3}$ \\
\hline
\end{tabular}

\subsection{Conclusion}

In both particular cases we have studied, we can conclude that even if the solutions considered do not satisfy the assumptions of Theorem 1.1 of [15], the rate of convergence is at least 3/4. Moreover, it seems that the error converges to a constant when $\varepsilon$ tends to zero. Then we can think that this rate could be the best. However, the perturbation due to the periodic boundary conditions does not allow us to consider longer simulations.

\section{The COUPLing EFFECTS}

In this section we will consider the case of two waves propagating in two opposite directions. We wonder what happens if we consider a solution of (BL) having the form

$$
\Phi(x, y, t)=f\left(x-t, \varepsilon^{1 / 2} y, \varepsilon t / 2\right)-g\left(x+t, \varepsilon^{1 / 2} y, \varepsilon t / 2\right)+\rho_{\varepsilon}\left(x, \varepsilon^{1 / 2} y, t\right),
$$

where

$$
\sup _{t \in\left[0,2 T_{0} / \varepsilon\right]}\left\|\rho_{\varepsilon t}(\cdot, \cdot, t)\right\|_{L^{2}\left(\mathbb{R}^{2}\right)}^{2}+\left\|\rho_{\varepsilon x}(\cdot, \cdot, t)\right\|_{L^{2}\left(\mathbb{R}^{2}\right)}^{2} \underset{\varepsilon \rightarrow 0}{\longrightarrow} 0 .
$$

In this case we can expect $u_{1}=f_{X}$ and $u_{2}=g_{\bar{X}}$ to be solutions of the following uncoupled system of KP equations

$$
\left\{\begin{array}{l}
\left(u_{1 T}-(a-b) u_{1 X X X}+3 u_{1} u_{1 X}\right)_{X}+u_{1 Y Y}=0, \\
\left(u_{2 T}+(a-b) u_{2 X X X}+3 u_{2} u_{2} \bar{X}\right) \bar{X}-u_{2 Y Y}=0,
\end{array}\right.
$$

where $\bar{X}=x+t$. Let remark that if $a-b>0$ (resp. $a-b<0$ ) we have two KP-I (resp. KP-II) equations. In [15], there is no theorical result about this question, then we will look for the rate of convergence we can expect.

Let

$$
\begin{aligned}
& u_{1}(X, Y, T)=A_{1} \operatorname{sech}^{2}\left[k_{1} X+k_{2} Y-\omega_{1} T\right], \\
& u_{2}(X, Y, T)=A_{2} \operatorname{sech}^{2}\left[k_{1} X+k_{2} Y-\omega_{2} T\right],
\end{aligned}
$$

be respectively two line solitons of the two equations of (25) where $A_{1}=-4 k_{1}^{2}(a-b)=-A_{2}$ and $\omega_{1}=$ $\frac{k_{2}^{2}-4 k_{1}^{4}(a-b)}{k_{1}}=-\omega_{2}$. The corresponding periodicised initial data are

$$
\begin{aligned}
& \Phi_{0}(x, y)=2 \frac{A_{1}}{k_{1}}\left[\tanh \left(k_{1} x+k_{2} \varepsilon^{1 / 2} y\right)-\frac{1}{k_{1} L} \tanh \left(k_{1} L\right)\left(k_{1} x+k_{2} \varepsilon^{1 / 2} y\right)\right] \\
& \Phi_{1}(x, y)=0 .
\end{aligned}
$$

Hereafter, we compare the numerical solution of the Benney-Luke equation to $-u_{1}\left(x-t, \varepsilon^{1 / 2} y, \varepsilon t / 2\right)-u_{2}(x+$ $\left.t, \varepsilon^{1 / 2} y, \varepsilon t / 2\right)$, thus we plot the evolution of $\left\|-\Phi_{t}+u_{1}+u_{2}\right\|_{l \infty}$ when $\varepsilon$ decrease. Precisely, the comparisons are made in the KP-I case where $a=2 / 3, b=1 / 3, k_{1}=1$ and $k_{2}=10$. We sum up in the next tabular the data for our experiments. 


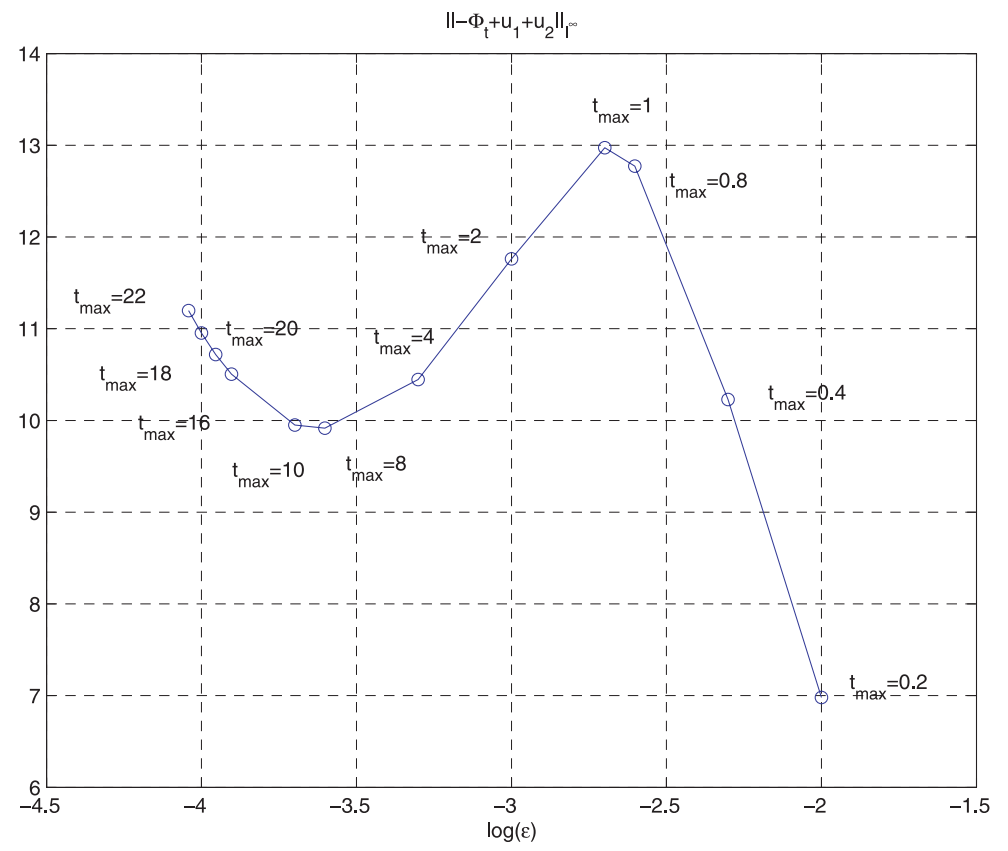

FigURE 20. KP-I $\sup _{\left[0,2 T_{0} / \varepsilon\right]} \frac{\left\|-\Phi_{t}+u_{1}+u_{2}\right\|_{l \infty}}{\varepsilon^{3 / 4}}: 256 \times 256$ points, $\Delta t=0.005$.

\begin{tabular}{|c|c|c|c|c|c|c|}
\hline$\varepsilon$ & 0.01 & 0.005 & 0.0025 & 0.002 & 0.001 & 0.0005 \\
\hline$L$ & $4 \pi$ & $4 \pi$ & $4 \pi$ & $4 \pi$ & $4 \pi$ & $4 \pi$ \\
\hline$M_{\varepsilon}$ & 12.56 & 17.78 & 25.13 & 28.10 & 39.74 & 56.20 \\
\hline$\Delta x$ & $1.96 \times 10^{-1}$ & $1.96 \times 10^{-1}$ & $1.96 \times 10^{-1}$ & $1.96 \times 10^{-1}$ & $1.96 \times 10^{-1}$ & $1.96 \times 10^{-1}$ \\
\hline$\Delta y$ & $4.91 \times 10^{-2}$ & $6.94 \times 10^{-2}$ & $9.82 \times 10^{-2}$ & $1.09 \times 10^{-1}$ & $1.55 \times 10^{-1}$ & $2.19 \times 10^{-1}$ \\
\hline$t_{\max }$ & 0.2 & 0.4 & 0.8 & 1 & 2 & 4 \\
\hline $\mathcal{E} r r_{\infty}$ & $2.21 \times 10^{-1}$ & $1.92 \times 10^{-1}$ & $1.43 \times 10^{-1}$ & $1.23 \times 10^{-1}$ & $6.61 \times 10^{-2}$ & $3.49 \times 10^{-2}$ \\
\hline
\end{tabular}

\begin{tabular}{|c|c|c|c|c|c|c|}
\hline$\varepsilon$ & 0.00025 & 0.0002 & 0.000125 & $1.11 .10^{-4}$ & 0.0001 & $9.09 .10^{-5}$ \\
\hline$L$ & $4 \pi$ & $4 \pi$ & $4 \pi$ & $4 \pi$ & $4 \pi$ & $4 \pi$ \\
\hline$M_{\varepsilon}$ & 79.48 & 88.86 & 112.4 & 119.2 & 125.6 & 131.8 \\
\hline$\Delta x$ & $1.96 \times 10^{-1}$ & $1.96 \times 10^{-1}$ & $1.96 \times 10^{-1}$ & $1.96 \times 10^{-1}$ & $1.96 \times 10^{-1}$ & $1.96 \times 10^{-1}$ \\
\hline$\Delta y$ & $3.10 \times 10^{-1}$ & $3.47 \times 10^{-1}$ & $4.39 \times 10^{-1}$ & $4.66 \times 10^{-1}$ & $4.91 \times 10^{-1}$ & $5.15 \times 10^{-1}$ \\
\hline$t_{\max }$ & 8 & 10 & 16 & 18 & 20 & 22 \\
\hline $\mathcal{E} r r_{\infty}$ & $1.97 \times 10^{-2}$ & $1.67 \times 10^{-2}$ & $1.24 \times 10^{-2}$ & $1.16 \times 10^{-2}$ & $1.09 \times 10^{-2}$ & $1.04 \times 10^{-2}$ \\
\hline
\end{tabular}

In Figures 21-28, we show the evolution of the shape of $-\Phi_{t}\left(t_{\max }\right)$ for some $\varepsilon$ in order to well understand Figure 20. In this figure, we point out the interaction phenomena between the waves. Indeed, the error increases when the wave are close $\left(\varepsilon \in\left[10^{-3} ; 10^{-2}\right]\right)$ and then it decreases when they separate $\left(\varepsilon \in\left[5 \times 10^{-4} ; 10^{-3}\right]\right)$. Since we simulate the periodic case, another wave begin to interact with the first one, thus the error increases again. This is an illustration of the limits of the approximation of Benney-Luke by a uncoupled system of two KP equations. In [4] Ben Youssef and Lannes confirm this fact for a general hyperbolic system by showing that the approximation obtained by a coupled system of KP equations seems to be better. However, the decrease of the error after separation of the waves leads us to think that if we do not have considered periodic boundary conditions, we should have obtained the same behaviour as the one-wave case, that is the rate of convergence 


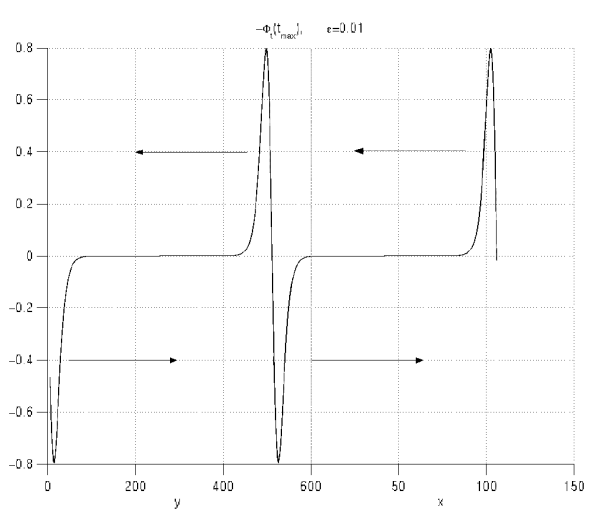

Figure 21. $-\Phi_{t}\left(t_{\max }\right), \varepsilon=$ $0.01, t_{\max }=0.2$.

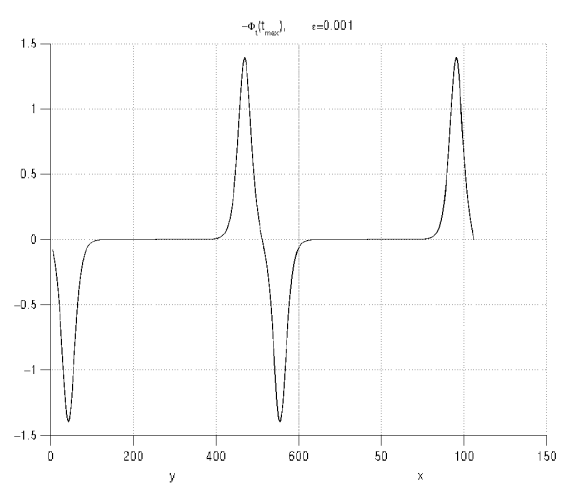

Figure 23. $-\Phi_{t}\left(t_{\max }\right)$, $\varepsilon=0.001, t_{\max }=2$.

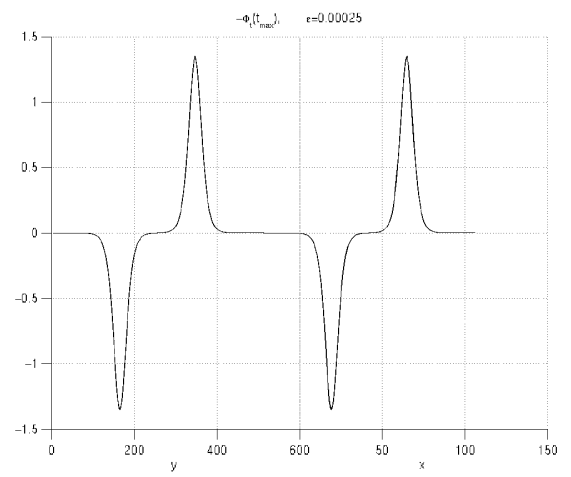

FiguRE 25. $-\Phi_{t}\left(t_{\max }\right)$, $\varepsilon=0.00025, t_{\max }=8$.

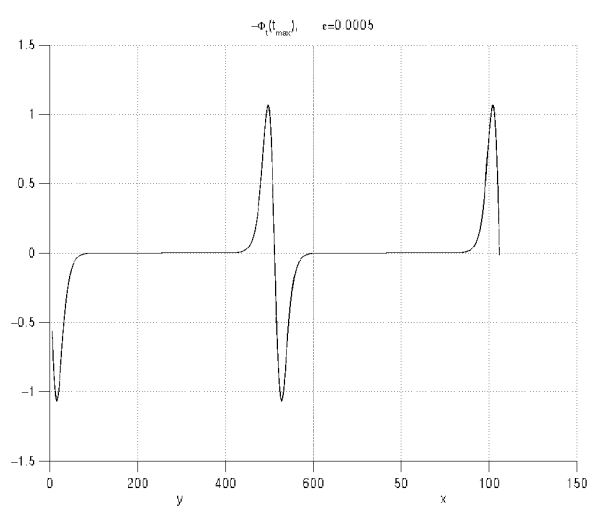

Figure 22. $-\Phi_{t}\left(t_{\max }\right), \varepsilon=$ $0.005, t_{\max }=0.4$.

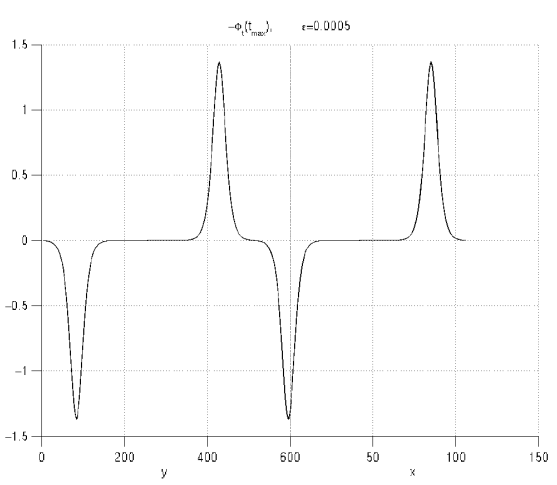

Figure 24. $-\Phi_{t}\left(t_{\max }\right)$, $\varepsilon=0.0005, t_{\max }=4$.

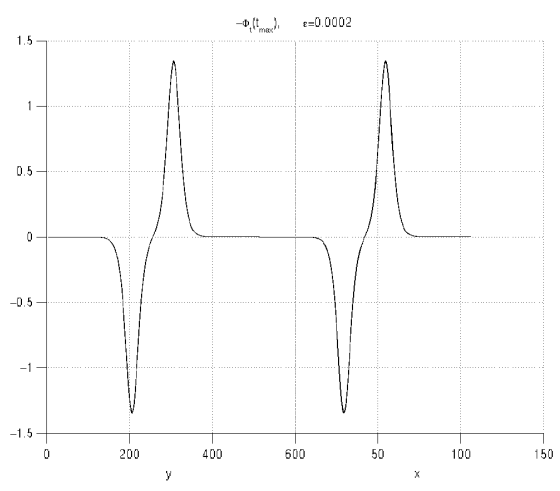

Figure 26. $-\Phi_{t}\left(t_{\max }\right)$, $\varepsilon=0.0002, t_{\max }=10$. 


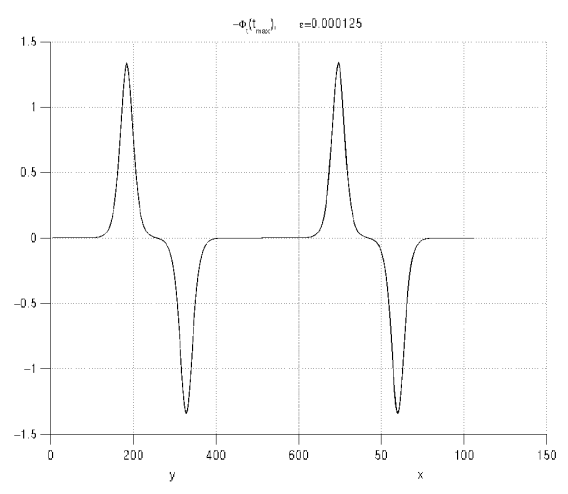

FIGURE 27. $-\Phi_{t}\left(t_{\max }\right)$, $\varepsilon=0.000125, t_{\max }=16$.

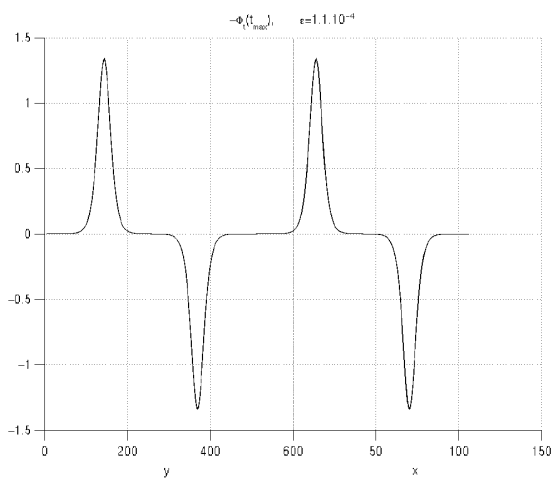

Figure 28. $-\Phi_{t}\left(t_{\max }\right)$, $\varepsilon=1.1110^{-4}, t_{\max }=18$.

could be the same. An interesting work would be to show numerically that there is a real improvement considering the coupling mode in the KP equations when the waves interact. It would seem to be interesting to use a symmetric decomposition in our numerical scheme, but it is not so relevant because such a choice privileges the decoupling effects.

Acknowledgements. We want to thank the referees for their constructive remarks.

\section{REFERENCES}

[1] M.J. Ablowitz and H. Segur, On the evolution of packets of water waves. J. Fluid Mech. 92 (1979) 691-715.

[2] J.C. Alexander, R.L. Pego and R.L. Sachs, On the transverse instability of solitary waves in the Kadomtsev-Petviashvili equation. Phys. Lett. A 226 (1997) 187-192.

[3] W. Ben Youssef and T. Colin, Rigorous derivation of Korteweg-de Vries-type systems from a general class of nonlinear hyperbolic systems. ESAIM: M2AN 34 (2000) 873-911.

[4] W. Ben Youssef and D. Lannes, The long wave limit for a general class of 2D quasilinear hyperbolic problems. Comm. Partial Differ. Equations 27 (2002) 979-1020.

[5] D.J. Benney and J.C. Luke, On the interactions of permanent waves of finite amplitude. J. Math. Phys. 43 (1964) $309-313$.

[6] K.M. Berger and P.A. Milewski, The generation and evolution of lump solitary waves in surface-tension-dominated flows. SIAM J. Appl. Math. 61 (2002) 731-750 (electronic).

[7] J.L. Bona, T. Colin and D. Lannes, Long wave approximations for water waves. Preprint Université de Bordeaux I, U-03-22 (2003).

[8] W. Craig, An existence theory for water waves and the Boussinesq and Korteweg-de Vries scaling limits. Comm. Partial Differ. Equations 10 (1985) 787-1003.

[9] T. Gallay and G. Schneider, KP description of unidirectional long waves. The model case. Proc. Roy. Soc. Edinburgh Sect. A 131 (2001) 885-898.

[10] T. Kano and T. Nishida, A mathematical justification for Korteweg-de Vries equation and Boussinesq equation of water surface waves. Osaka J. Math. 23 (1986) 389-413.

[11] D. Lannes, Consistency of the KP approximation. Discrete Contin. Dyn. Syst. (suppl.) (2003) 517-525. Dynam. Syst. Differ. equations (Wilmington, NC, 2002).

[12] P.A. Milewski and J.B. Keller, Three-dimensional water waves. Stud. Appl. Math. 97 (1996) 149-166.

[13] P.A. Milewski and E.G. Tabak, A pseudospectral procedure for the solution of nonlinear wave equations with examples from free-surface flows. SIAM J. Sci. Comput. 21 (1999) 1102-1114 (electronic).

[14] L. Paumond, Towards a rigorous derivation of the fifth order KP equation. Submitted for publication (2002).

[15] L. Paumond, A rigorous link between KP and a Benney-Luke equation. Differential Integral Equations 16 (2003) 1039-1064.

[16] R.L. Pego and J.R. Quintero, Two-dimensional solitary waves for a Benney-Luke equation. Physica D 132 (1999) $476-496$.

[17] G. Schneider and C.E. Wayne, The long-wave limit for the water wave problem. I. The case of zero surface tension. Comm. Pure Appl. Math. 53 (2000) 1475-1535. 